\title{
ANÁLISE DE TENDÊNCIAS E EXTREMOS PLUVIOMÉTRICOS NA REGIÃO HIDROGRÁFICA DO RIO SÃO FRANCISCO
}

\author{
Mariana Mendes Silva ${ }^{1}$ \\ Vanderlei de Oliveira Ferreira ${ }^{2}$ \\ Dane Marques de Ávila ${ }^{3}$
}

Resumo: Este artigo relata resultados de pesquisa destinada à detecção e análise de tendências e extremos pluviométricos na Região Hidrográfica do Rio São Francisco. Para tanto, foram utilizados dados de 124 postos pluviométricos da Agência Nacional de Águas (ANA), cujo período analisado foi de 1976 a 2015 (40 anos). Para a detecção e análise das tendências pluviométricas foram aplicadas as ferramentas estatísticas Regressão Linear, Teste de Mann-Kendall e Teste do Sinal. Para identificar e avaliar a frequência e a intensidade de eventos extremos foram utilizados o Standardized Precipitation Index - SPI (ou Índice de Precipitação Padronizada - IPP) e o Rainfall Anomaly Index - RAI (ou Índice de Anomalia de Chuva - IAC). Os resultados mostraram tendência à diminuição das chuvas na área estudada, além da maior possibilidade de ocorrências de extremos positivos e negativos nas classes próximas à normalidade.

Palavras-chave: Mudanças Climáticas; Tendências Pluviométricas; Extremos Pluviométricos; Região do São Francisco.

\section{ANALYSIS OF PLUVIOMETRIC TRENDS AND EXTREMES IN THE SÃO FRANCISCO RIVER HYDROGRAPHIC REGION}

\begin{abstract}
This article reports research results intended at detecting and analyzing pluviometric trends and extremes in the São Francisco River Hydrographic Region. For this purpose, were used data from 124 pluviometric stations of the National Water Agency (ANA), whose period analyzed was from 1976 to 2015 (40 years). For the detection and analysis of the pluviometric trends the following statistical tools were applied Linear Regression, Mann-Kendall Test and Signal Test. To identify and evaluate the frequency and intensity of extreme events were used the Standardized Precipitation Index - SPI and the Rainfall Anomaly Index - RAI. The results demonstrate tendency to decrease rainfall in the study area, in addition to the greater possibility of occurrences of positive and negative extremes in the classes close to normality.
\end{abstract}

Keywords: Climate Changes; Pluviometric Trends; Pluviometric Extremes; São Francisco Region.

\footnotetext{
${ }^{1}$ Doutora em Geografia pelo Programa de Pós-graduação em Geografia do Instituto de Geografia da Universidade Federal de Uberlândia - UFU. Email: marianamendes_01@yahoo.com.br

${ }^{2}$ Professor associado II da Universidade Federal de Uberlândia (UFU). Email: vanderlei.ferreira@ufu.br

${ }^{3}$ Professor do Instituto Federal do Triângulo Mineiro - Campus Ituiutaba. Email: danemarques@iftm.edu.br
} 


\section{INTRODUÇÃO}

Relatórios do Intergovernmental Panel on Climate Change - IPCC (2012, 2013, 2014) e pesquisas realizadas por renomadas instituições do mundo inteiro indicam evidências concretas sobre o aumento da temperatura no sistema Terra-Atmosfera e, consequentemente, um quadro de mudanças climáticas que atinge todas as escalas. Em tal contexto, o ciclo hidrológico é sistemicamente modificado. A quantidade e distribuição da fonte primária de água doce terrestre, as chuvas, são inevitavelmente alteradas.

Ainda que estudos voltados para as mudanças climáticas mostrem aumento na média da temperatura global e seus impactos para a natureza e as populações, a temática se mostra ainda complexa diante das limitações dos modelos e ferramentas matemáticas e computacionais, principalmente quando aplicadas em âmbitos regional e local. Experiências de modelagem e utilização de instrumentos matemáticos complexos têm apresentado resultados mais satisfatórios nas escalas global e zonal do que nas escalas regional e local, pelo fato da necessidade de melhorias na resolução espacial e também devido ao custo computacional.

Neste sentido, é necessário buscar alternativas para melhorar o tratamento das mudanças climáticas em escala regional/local com o intuito de se alcançar melhores resultados sem a necessidade de grandes investimentos. Logo, o objetivo da pesquisa relatada neste artigo é estudar as tendências e os eventos de extremos pluviométricos na Região Hidrográfica do Rio São Francisco aplicando as seguintes ferramentas: Regressão Linear, Teste de Mann-Kendall e Teste do Sinal - para detecção de tendências; e Standardized Precipitation Index - SPI (ou Índice de Precipitação Padronizada - IPP) e o Rainfall Anomaly Index - RAI (ou Índice de Anomalia de Chuva - IAC) - para identificação de extremos.

A área de estudo selecionada foi a Região Hidrográfica do Rio São Francisco, dada sua relevância nos contextos nacional e regional brasileiro, pela mesma possuir um desequilíbrio entre oferta e demanda dos recursos hídricos em diversas subbacias (conflitos), por apresentar especificidades fisiográficas significativas e devido aos sistemas atmosféricos dinâmicos que atuam diferenciadamente ao longo desta região hidrográfica, resultando em condições particulares de clima, solo, vegetação e também de atividades econômicas.

\section{LOCALIZAÇÃO DA ÁREA DE ESTUDO}

O Brasil possui 12 regiões hidrográficas. Uma delas é a Região Hidrográfica do Rio São Francisco (Figura 1), que corresponde a 8\% do território nacional. Ela possui uma área de drenagem de aproximadamente $640.000 \mathrm{~km}^{2}$, distribuída nos estados da Bahia/BA, Minas Gerais/MG, Pernambuco/PE, Alagoas/AL, Sergipe/SE, Goiás/GO e Distrito Federal/DF (sítio eletrônico do Comitê da Bacia Hidrográfica do Rio São Francisco - CBHSF, s.d., disponível em: <http://cbhsaofrancisco.org.br/a-bacia/>). A mesma foi dividida em dez (10) sub-bacias, segundo a Agência Nacional de Águas ANA. São elas: Alto São Francisco até Três Marias (40), das Velhas (41), Paracatu e outros (42), Urucuia (43), Verde Grande (44), Corrente e outros (45), Grande e outros (46), Salitre e outros (47), Pajéu e outros (48), São Francisco a jusante de Pajéu (49).

Segundo o CBHSF (s.d.), o Rio São Francisco tem aproximadamente 2.700 km de extensão e nasce no Parque Nacional da Serra da Canastra no sudoeste do estado de Minas Gerais, escoa no sentido sul-norte pelos estados da Bahia e Pernambuco, quando altera seu curso para leste, chegando ao Oceano Atlântico através da divisa 
entre Alagoas e Sergipe. Em seu trajeto corta três biomas: a Caatinga na região nordeste da Bahia; o Cerrado entre o sudoeste de Minas Gerais e norte da Bahia; e a Mata Atlântica na região da Serra da Canastra.

Figura 1. Localização da Região Hidrográfica do Rio São Francisco

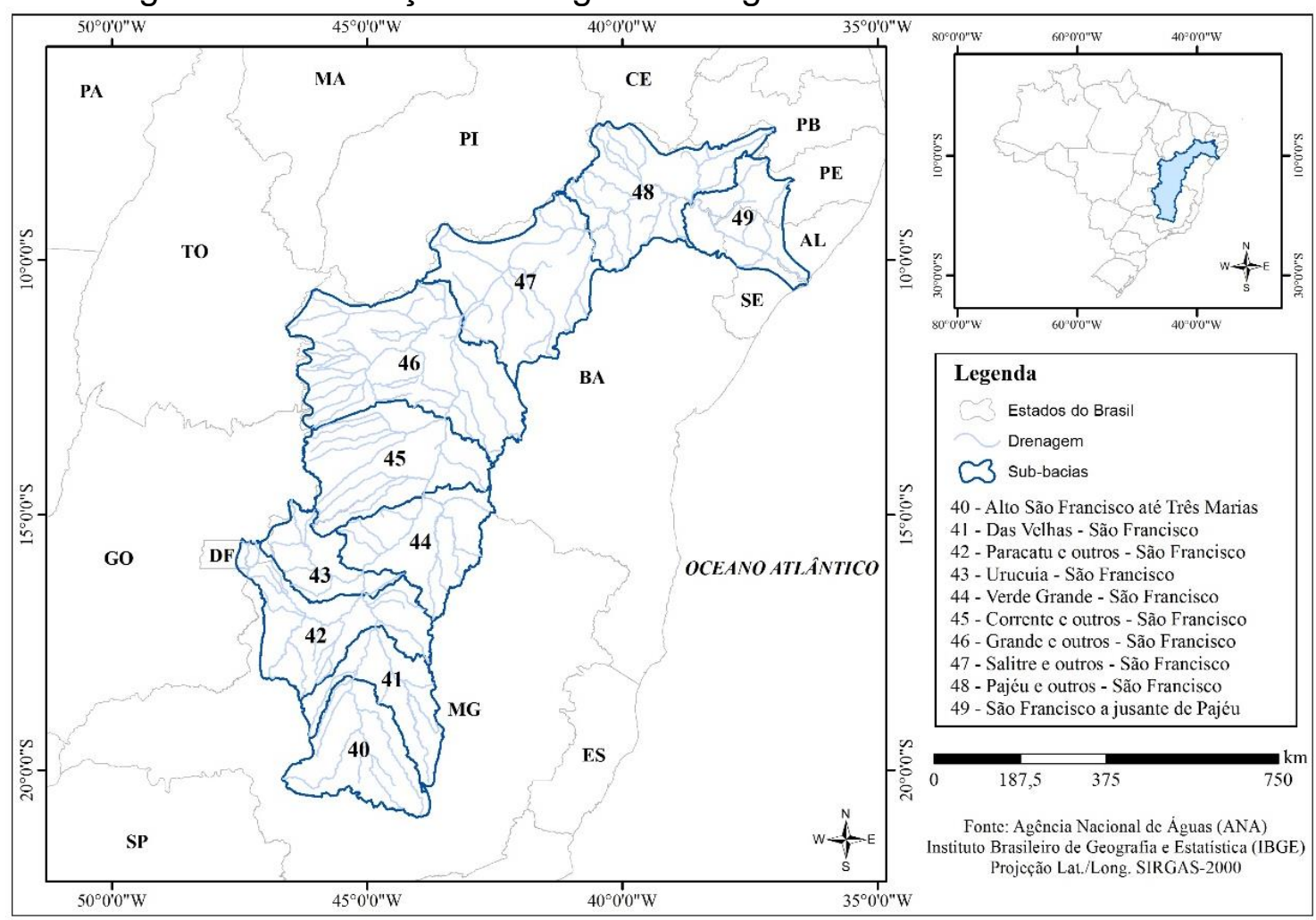

Fonte: ANA (2017); IBGE (2017).

Organização: Os autores.

\section{PROCEDIMENTOS METODOLÓGICOS}

\section{SELEÇÃO DOS POSTOS PLUVIOMÉTRICOS}

Para este artigo foram selecionados dados de 124 postos pluviométricos da rede de monitoramento da ANA (Quadro 1 e Figura 2), considerados suficientes e viáveis do ponto de vista da aplicação das ferramentas estatísticas e geração de resultados.

No que diz respeito às eventuais ausências de dados, optou-se por preencher falhas com o valor da média mensal, calculado a partir de todos os dados do específico mês e posto pluviométrico, isto é, se não havia um dado no mês de janeiro (por exemplo) de um ano qualquer, essa lacuna foi preenchida com a média mensal de janeiro calculada no período de 1976-2015 do respectivo posto pluviométrico. 
Quadro 1. Postos Pluviométricos adotados na pesquisa

\begin{tabular}{|c|c|c|}
\hline Sub-bacia & Código dos Postos & Total de Postos \\
\hline $\begin{array}{l}40 \text { - Alto São } \\
\text { Francisco até } \\
\text { Três Marias }\end{array}$ & $\begin{array}{l}1845002,1845004,1944004,1944007,1944010 \\
1944011,1944026,1944031,1944049,1945002, \\
1945008,1945019,1945035,1946000,1946009 \\
2043005,2043013,2044002,2044003,2044007 \\
2044008,2044009,2044012,2044016,2044019 \\
2044020,2044021,2044024,2044026,2045001 \\
2045002,2045010,2045011,2045012,2045013 \\
2046007,2046013\end{array}$ & 37 \\
\hline 41 - das Velhas & 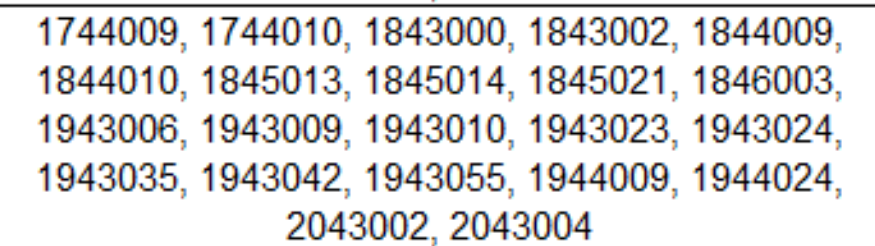 & 22 \\
\hline $\begin{array}{l}42 \text { - Paracatu e } \\
\text { outros }\end{array}$ & $\begin{array}{c}1644028,1645009,1645013,1646000,1646003 \\
1745001,1746001,1746002,1746007,1747005 \\
1846015,1846016\end{array}$ & 12 \\
\hline 43 - Urucuia & $\begin{array}{c}1546000,1546005, \begin{array}{l}1644027,1645000,1645002, \\
1645005\end{array} \\
\end{array}$ & 6 \\
\hline $\begin{array}{l}44 \text { - Verde } \\
\text { Grande }\end{array}$ & $1443001,1543002,1543013,1544012,1544019$ & 5 \\
\hline $\begin{array}{l}45 \text { - Corrente e } \\
\text { outros }\end{array}$ & $\begin{array}{l}\text { 1344002, 1344014, 1344015, 1443002, } 1444000 \\
1444001,1444003,1444004,1444005,1444017\end{array}$ & 10 \\
\hline $\begin{array}{l}46 \text { - Grande e } \\
\text { outros }\end{array}$ & $\begin{array}{l}1143010,1144005,1144008,1144014,1145004, \\
1145013,1145014,1242015,1242016,1243000, \\
1243011,1244011,1244019,1245005,1245007\end{array}$ & 15 \\
\hline $\begin{array}{l}47 \text { - Salitre e } \\
\text { outros }\end{array}$ & $940018,940028,1142017$ & 3 \\
\hline $\begin{array}{l}48 \text { - Pajéu e } \\
\text { outros }\end{array}$ & $\begin{array}{c}737023,737027,838000,838002,838004,839009, \\
839014,840010,840015,940025\end{array}$ & 10 \\
\hline $\begin{array}{l}49 \text { - São } \\
\text { Francisco a } \\
\text { jusante de } \\
\text { Pajéu }\end{array}$ & $837038,936076,937013,1036005$ & 4 \\
\hline
\end{tabular}

Fonte: ANA (2017).

Fonte: Organizado pelos autores 
Figura 2. Localização dos 124 Postos Pluviométricos adotados na pesquisa

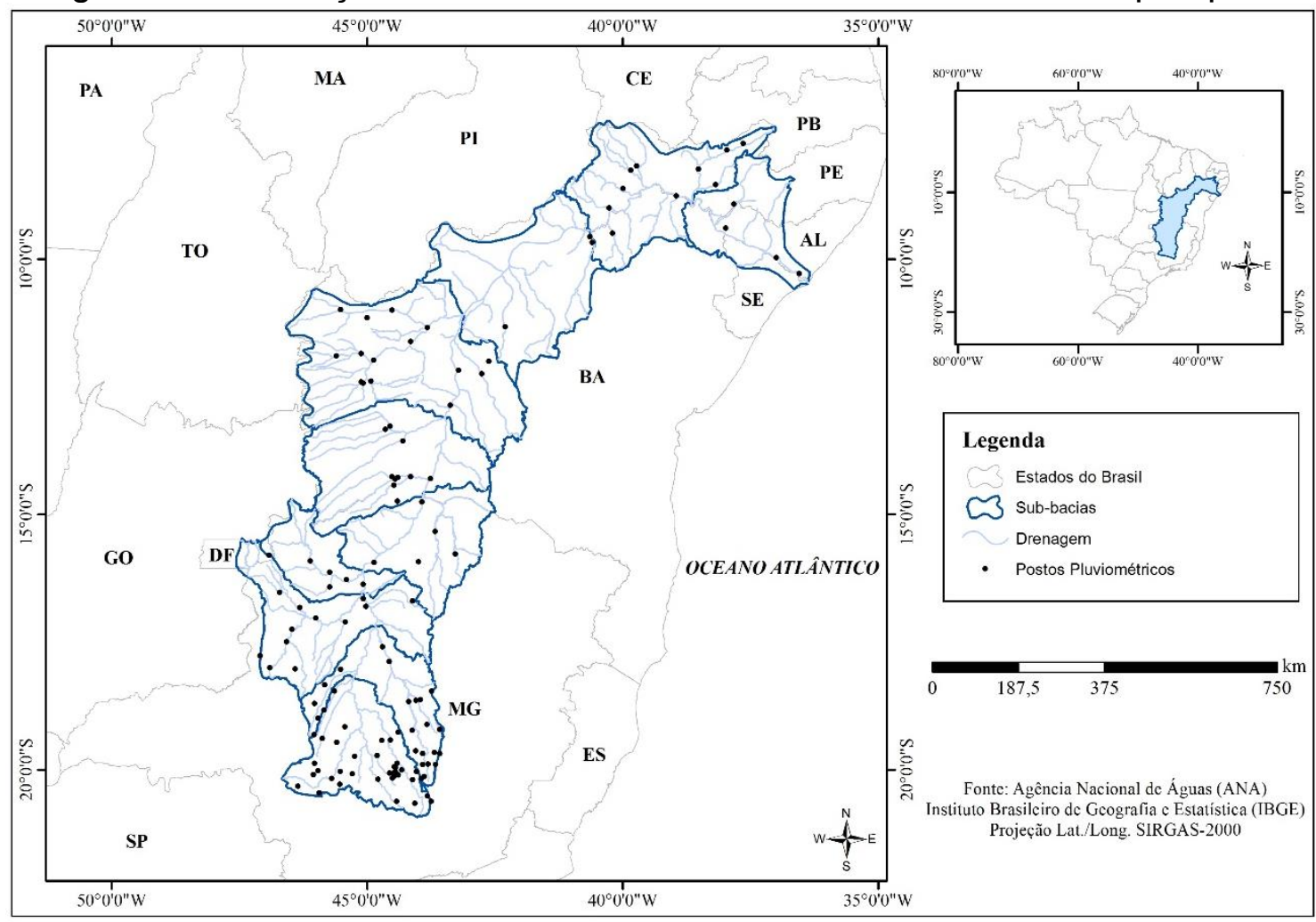

Fonte: ANA (2017); IBGE (2017).

Fonte: Organizado pelos autores

\section{INSTRUMENTOS APLICADOS À DETECÇÃO E AVALIAÇÃO DE TENDÊNCIAS PLUVIOMÉTRICAS}

Segundo Morettin e Toloi (2006), a tendência de uma série temporal indica seu comportamento de longo prazo, isto é, se ela cresce, decresce ou permanece estável. Back (2001, p. 718) define tendência em uma série temporal "como uma mudança sistemática e contínua em qualquer parâmetro de uma dada amostra, excluindo-se mudanças periódicas ou quase periódicas". Logo, neste trabalho, as tendências foram definidas como modificações graduais e contínuas do comportamento da pluviometria e que podem ser de aumento ou redução, provocadas por diferentes causas.

Como na literatura há várias ferramentas e métodos que analisam o comportamento de séries históricas e estimam valores futuros com diferentes perspectivas, escolher as que melhor descrevem as tendências não é uma tarefa fácil. Por isso, é necessário o conhecimento de tais instrumentos e métodos para que o resultado seja eficaz. Neste sentido, após leituras sobre o assunto, foram selecionados e empregues neste estudo a Regressão Linear, o Teste de MannKendall e o Teste do Sinal.

A análise de regressão tem por intenção descrever por meio de um modelo matemático, a relação entre duas variáveis, baseando-se nas $\boldsymbol{n}$ observações das mesmas. Neste sentido, a reta de regressão serve para resumir a relação entre as variáveis e para fazer previsões a partir dos dados. Então, primeiramente é estabelecida a equação da reta por meio da função $\boldsymbol{Y}=\boldsymbol{a} \boldsymbol{X}+\boldsymbol{b}$ (sendo $\boldsymbol{Y}$ a variável dependente, $\boldsymbol{X}$ a variável independente e $\boldsymbol{a}$ e $\boldsymbol{b}$ os parâmetros), cuja reta resultante representa visualmente a correlação das variáveis, que neste estudo são: pluviometria 
e tempo. Para definir a equação da reta, foram calculados os valores dos parâmetros utilizando a seguinte fórmula:

$$
\begin{array}{r}
a=\frac{n \Sigma x_{i} y i-\Sigma x_{i} \Sigma y i}{n \Sigma x_{i}^{2}-\left(\Sigma x_{i}\right)^{2}} \\
b=\bar{y}-a \bar{x}
\end{array}
$$

Onde: $\boldsymbol{n}$ é o número de observações; $\overline{\boldsymbol{x}}$ é a média dos valores $x_{i}\left(\bar{x}=\frac{\sum x_{i}}{n}\right)$; e $\overline{\boldsymbol{y}}$ é a

$$
\text { média dos valores } y i\left(\bar{y}=\frac{\Sigma y i}{n}\right) \text {. }
$$

Como parte da definição das retas de regressão, o coeficiente angular de cada uma das retas (parâmetro a) corresponde ao valor da tangente do ângulo formado entre a reta de regressão e o eixo " $X$ ", o que determina a inclinação da reta. $O$ parâmetro a permite observar o quanto a inclinação da reta é expressiva ou não, demonstrando se há tendência e o quanto ela é significante ou não. Tais cálculos foram processados no Microsoft Office Excel 2016;

Quanto ao Teste de Mann-Kendall "é um método robusto, sequencial e não paramétrico utilizado para determinar se determinada série de dados possui uma tendência temporal de alteração estatisticamente significativa" (SALVIANO et al., 2016, p. 65). Diante disso, para aplicar esta ferramenta, primeiramente é necessário saber se a série temporal da pluviometria é independente e identicamente distribuída. Assim, para comprovar que os índices pluviométricos são serialmente independentes, foram testadas as hipóteses: $H_{0}=$ as observações da série são independentes e identicamente distribuídas - não há tendência; e $H_{1}=$ as observações da série possuem tendência monotônica no tempo - há tendência. No ponto de vista do $H_{0}$, a estatística do teste é dada por:

$$
\begin{gathered}
S=\sum_{k=1}^{n-1} \sum_{j=k+1}^{n} \operatorname{sing}\left(x_{j}-x_{k}\right) \\
\operatorname{sign}=\left\{\begin{array}{c}
1, \text { se } x>0 \\
0, \text { se } x=0 \\
-1, \text { se } x<0
\end{array}\right.
\end{gathered}
$$

Para demonstrar que $S$ é normalmente distribuída,

$$
\begin{gathered}
S \sim N\left(\mu, \sigma^{2}\right) \operatorname{com} \mu=0 \\
\sigma^{2}=\frac{n^{e}(n-1)(2 n+5)-\sum_{j-1}^{P} t_{j}\left(t_{j}-1\right)\left(2 t_{j}+5\right)}{18}
\end{gathered}
$$

Em que $n$ é o número de observações; $P$ é o número de grupos de observações iguais e $t_{j}$ é o numero de observações iguais no grupo $j$. Se no caso, o número de observações for superior a 30, a estatística do teste é dada por: 


$$
Z=\left\{\begin{array}{l}
\frac{S-1}{\sigma}, \text { se } S>0 \\
0, \text { se } S=0 \\
\frac{S+1}{\sigma}, \text { se } S<0
\end{array}\right.
$$

Para um teste bilateral, não se pode rejeitar a hipótese nula $H_{0}$ para um dado nível de significância $\alpha$, se para o quantil $Z_{\alpha / 2}$ de uma distribuição normal padrão temos $|Z| \leq Z_{\alpha / 2}$. O teste de Mann-Kendall foi executado no Action Stat 3, um software estatístico desenvolvido pelo Portal Action mantido pela empresa "Estatcamp Consultoria Estatística e Qualidade". Este software se conecta com a interface do Microsoft Office Excel. Os resultados dos testes foram apreciados juntamente com a Análise de Regressão e também com os cálculos do Coeficiente Angular das Retas de Regressão Linear.

Segundo Ferreira (2012, p. 321), "o Teste do Sinal é uma alternativa simples para detectar tendências em séries temporais, embora não recomendado como ferramenta única". Por isso, não se pode inferir qualquer suposição tendo como base apenas sua aplicação. Ele é calculado pelas diferenças entre os pares de observações, sendo necessário, primeiramente, dividir a série temporal em duas subséries de tamanhos iguais. Como as séries pluviométricas utilizadas nesta pesquisa são de 40 anos (1976-2015), elas foram divididas em duas partes com tamanhos idênticos: $\boldsymbol{x}_{\boldsymbol{i}}(1976-1995)$ e $\boldsymbol{y}_{\boldsymbol{i}}(1996-2015)$.

Em seguida, realiza-se o cálculo da diferença $(\boldsymbol{D} \boldsymbol{i})$ para cada par de observações $\left(\boldsymbol{D} \boldsymbol{i}=\boldsymbol{\chi}_{\boldsymbol{i}}-\boldsymbol{y}_{\boldsymbol{i}}\right)$. Assim, se $\boldsymbol{D} \boldsymbol{i}$ é maior que zero, ao par é atribuído um sinal de positivo (+); se $\boldsymbol{D} \boldsymbol{i}$ é menor que zero, ele recebe um sinal de negativo (-); e se $\boldsymbol{D} \boldsymbol{i}$ é igual a zero, exclui-se o par de observações na contabilização entre a quantidade de números de sinais positivos e negativos e a amostra é reduzida.

Após o cálculo de $\boldsymbol{D} \boldsymbol{i}$, compara-se a quantidade de resultados com sinais negativos e positivos. Se a quantidade de negativos prevalecer, assume-se que está havendo tendência de aumento da pluviometria na série analisada; se predomina a quantidade de positivos, admite-se que há tendência de redução na série pluviométrica; e quando o número de sinais positivos foi igual ao número de sinais negativos, a tendência indicada é de manutenção do comportamento da variável. Vale lembrar que, na presente pesquisa, todo esse procedimento foi realizado no Microsoft Office Excel 2016.

Os métodos de detecção de tendências pluviométricas aqui referenciados foram aplicados por meio da adoção dos dados dos totais anuais e mensais de cada posto pluviométrico selecionado para o estudo na Região Hidrográfica do São Francisco.

\section{INSTRUMENTOS APLICADOS À IDENTIFICAÇÃO E AVALIAÇÃO DE EVENTOS DE EXTREMOS PLUVIOMÉTRICOS}

Conforme indica o próprio IPCC (2014), a variabilidade e os eventos climáticos extremos são muito importantes no contexto de tomada de decisões. Isto porque resultam em impactos significativos tanto para a sociedade quanto para o sistema natural. Neste sentido, estudar, identificar e avaliar os extremos pluviométricos é fundamental.

Então, além da utilização das ferramentas de detecção de tendências, já explicitadas anteriormente neste trabalho, foram aplicados métodos matemáticos para 
identificar e avaliar possíveis eventos extremos negativos e positivos nas séries de pluviometria. Para tanto, foram utilizados os cálculos dos chamados Standardized Precipitation Index (SPI) ou Índice Padronizado de Precipitação e Rainfall Anomaly Index (RAI) ou Índice de Anomalia de Chuva.

O SPI é aplicado em estudos sobre secas e extremos de chuvas. Quando criado, tinha como principal finalidade auxiliar na quantificação e no monitoramento de eventos de secas, mas na atualidade, ele tem sido empregado também no reconhecimento de episódios ligados ao excesso de pluviosidade (GARCIA et al., 2018). Para o cálculo do SPI é necessário um intervalo mínimo de 30 anos de dados de pluviosidade. Os dados são ajustados por meio da distribuição gama "que posteriormente é transformada em uma distribuição normal, que por definição, apresenta sua média com valor zero e variância unitária" (FERNANDES et al., EMBRAPA - Documentos 244, 2009).

A distribuição gama é definida pela função de densidade de probabilidade por meio da seguinte função:

$$
g(x)=\frac{1}{\Gamma(\alpha) \beta \alpha} x^{\alpha-1} e^{-\frac{\chi}{\beta}}
$$

Onde:

$\alpha>0$, parâmetro de forma (adimensional);

$\beta>0$, parâmetro de escala $(\mathrm{mm})$;

$x>0$, total de precipitação $(\mathrm{mm})$;

$\Gamma(x)$, função gama.

A função gama é calculada pela seguinte equação:

$$
\Gamma_{(\alpha)}=\int_{0}^{\infty} y^{\alpha-1} e^{-y} d y
$$

Para estimar os parâmetros $\alpha$ e $\beta$ - método da máxima verossimilhança - foram utilizadas as equações abaixo:

Onde:

$$
\begin{gathered}
\alpha=\frac{1}{4 A}\left(1+\sqrt{\frac{1+4 A}{3}}\right) \\
\beta=\frac{\bar{x}}{\alpha} \\
A=I_{n}(\bar{x})-\frac{1}{N} \sum_{i=1}^{N} \ln (x)
\end{gathered}
$$

$\bar{x}$, é a média aritmética da precipitação pluvial $(\mathrm{mm})$;

In, é o logaritmo neperiano;

$N$, é o número de observações de precipitação. 
Os resultados desses parâmetros são empregados para encontrar a probabilidade cumulativa de um evento de precipitação observado para uma escala de tempo mensal, utilizando a seguinte fórmula:

$$
G_{(x)}=\int_{0}^{x} g(x) d x=\frac{1}{\beta^{\alpha} \Gamma(\alpha)} \int_{0}^{\chi} \chi^{\alpha-1} e^{-\frac{x}{\beta}} d x
$$

Substituindo $t=\frac{\alpha}{\beta}$ na equação, a função gama incompleta fica:

$$
G_{(x)}=\frac{1}{\Gamma(\alpha)} \int_{0}^{x} t^{\alpha-1} e^{-t} d t
$$

Sob condição de que a função gama é indeterminada para $x=0$ e uma distribuição de pluviosidade pode conter zeros, a probabilidade cumulativa leva em consideração:

$$
H(x)=q+(1-q) G_{(x)}
$$

Sendo,

$H(x)$, distribuição de probabilidade cumulativa;

$q$, probabilidade de ocorrência de valores nulos (zeros);

$G_{(x)}$, distribuição cumulativa teórica.

Uma vez que $m$ é a quantidade de zeros da série de pluviometria, Thom (1966) aponta que $q$ pode ser estimado por $m / n+1$ e utiliza tabelas da função gama incompleta para determinar a probabilidade cumulativa $G_{(x)}$.

A distribuição dessa probabilidade cumulativa $G_{(x)}$ é alterada para uma variável aleatória normalizada (SPI) com média zero e desvio padrão 1.

\begin{abstract}
Essa é uma transformação equiprobabilística que Panofsky e Brier (1958) definiram como uma característica essencial para transformar uma variável de distribuição gama para uma variável com a distribuição normal, onde a probabilidade de ser menor que um valor dado será igual à probabilidade de ser menor que o valor correspondente da variável transformada. (FERNANDES et al., EMBRAPA - Documentos 244, p. 19-20).
\end{abstract}

Nessa perspectiva, os dados pluviométricos são ordenados em ordem crescente de magnitude de modo que os valores são baixos e no qual o tamanho da amostra é calculado pela seguinte equação:

$$
q=\frac{m}{n+1}
$$

Onde, $m$, é o número de ordem dos valores de zero em uma série de pluviometria; $n$, corresponde ao tamanho da amostra. 
O valor do (SPI) é estimado pelas equações desenvolvidas por Abramowitz \& Stegun (1965), que transformam a probabilidade cumulativa em uma distribuição normal a variável (SPI). Os valores de (SPI) são definidos da seguinte maneira:

$$
\begin{aligned}
& S P I=-\left(t-\frac{C_{0}+C_{1} t+C_{2} t^{2}}{1+d_{1} t+d_{2} t^{2}+d_{3} t^{3}}\right) \text { para } 0<H(x) \leq 0,5 \\
& S P I=+\left(t-\frac{C_{0}+C_{1} t+C_{2} t^{2}}{1+d_{1} t+d_{2} t^{2}+d_{3} t^{3}}\right) \text { para } 0,5<H(x) \leq 1
\end{aligned}
$$

Sendo $t$ determinado pelas equações:

$$
\begin{gathered}
t=\sqrt{\operatorname{In}\left[\frac{1}{(H(x))^{2}}\right]} \text { para } 0<H(x) \leq 0,5 \\
t=\sqrt{\operatorname{In}\left[\frac{1}{-1(H(x))^{2}}\right]} \text { para } 0,5<H(x) \leq 1
\end{gathered}
$$

Na presente pesquisa os coeficientes utilizados nas equações de determinação dos valores de $S P I$, são: $C_{0}=2,515517 ; C_{1}=0,802853 ; C_{2}=0,010328 ; d_{1}=1,432788$; $d_{2}=0,189269$; e $d_{3}=0,001308$. Com os valores do $S P I$ calculados para cada um dos dados pluviométricos os mesmos foram enquadrados nas categorias apresentadas no Quadro 2.

Quadro 2. Valores do Índice Padronizado de Precipitação (SPI) e categorias de seca ou umidade

\begin{tabular}{|c|c|}
\hline Valores do SPI & CATEGORIAS \\
\hline$\geq+2,00$ & Extremamente Úmido \\
\hline$+1,00 a+1,99$ & Muito Úmido \\
\hline$+0,50 a+0,99$ & Moderadamente Úmido \\
\hline$+0,49 a-0,49$ & Normal \\
\hline$-0,50 a-0,99$ & Moderadamente Seco \\
\hline$-1,00 a-1,99$ & Muito Seco \\
\hline$\leq-2,00$ & Extremamente Seco \\
\hline
\end{tabular}

Fonte: Mackee et al. (1993).

Outro índice utilizado neste trabalho para identificar e analisar anomalias pluviométricas na Região Hidrográfica do Rio São Francisco foi o RAl. De acordo com Rooy (1965), este índice é considerado de simples cálculo, dada a sua facilidade de procedimento computacional, e interpretação. Além disso, classifica e ordena as magnitudes das anomalias em positivas e negativas. O mesmo é calculado pelas seguintes fórmulas:

- Para meses ou anos com anomalias positivas: $R A I=3\left[\frac{(p-\bar{p})}{(\bar{m}-\bar{p})}\right]$

- Para meses ou anos com anomalias negativas: $R A I=-3\left[\frac{(p-\bar{p})}{(\bar{x}-\bar{p})}\right]$

Sendo, $p$, a pluviosidade observada $(\mathrm{mm})$; $\bar{p}$, a pluviosidade média $(\mathrm{mm})$; 
$\bar{m}$, a média dos dez valores mais altos de pluviosidade da série temporal e escala (mensal ou anual) utilizados;

$\bar{x}$, a média dos dez valores mais baixos de pluviosidade da série temporal e escala (mensal ou anual) utilizados.

No entanto, para saber se o valor da pluviosidade observada $(p)$ se encaixa na fórmula das anomalias positivas ou na equação das anomalias negativas, é necessário comparar a mesma com a pluviosidade média $(\bar{p})$, ou seja, se $p$ foi maior que $\bar{p}$, aplica-se a primeira equação e se $p$ foi menor que $\bar{p}$, emprega-se a segunda equação. Após a determinação do RAl para os valores de pluviometria, os mesmos são classificados nos grupos (Quadro 3):

Quadro 3. Valores do Índice de Anomalia de Chuva (RAI) e categorias de seca ou umidade

\begin{tabular}{|c|c|}
\hline Valores do RAI & CATEGORIAS \\
\hline$\geq+4,00$ & Extremamente Úmido \\
\hline$+2,00 \mathrm{a}+3,99$ & Muito Úmido \\
\hline$+1,99 \mathrm{a} 0$ & Úmido \\
\hline $0 \mathrm{a}-1,99$ & Seco \\
\hline$-2,00 \mathrm{a}-3,99$ & Muito Seco \\
\hline$\leq-4,00$ & Extremamente Seco \\
\hline
\end{tabular}

Tais ferramentas foram aplicadas no Microsoft Office Excel 2016 para os totais anuais e mensais de cada posto pluviométrico.

\section{ANÁLISE DAS TENDÊNCIAS PLUVIOMÉTRICAS}

As tendências pluviométricas são as modificações graduais (tanto de aumento quanto de redução) no comportamento das chuvas ao longo dos anos. Pelo fato de a Região Hidrográfica do Rio São Francisco apresentar significativa diversidade físiconatural, a aplicação de ferramentas estatísticas para detecção e análise de tendências dos índices pluviométricos é de significativa utilidade para o planejamento e gestão dos recursos naturais e das atividades humanas e também para auxiliar outros estudos sobre a temática. Como já citado, este estudo utilizou três ferramentas: Regressão Linear, Teste de Mann-Kendall e Teste do Sinal.

Numa visão geral, os resultados de tais testes confirmam que a maioria dos postos pluviométricos utilizados registrou alguma tendência na pluviometria (Quadro 4) e que a tendência predominante em todos os métodos indica diminuição dos índices pluviométricos, tanto no total anual quanto nos meses do ano.

Vale expor que a região hidrográfica possui uma porcentagem de ocorrências de tendência negativa nos meses do ano de aproximadamente $66 \%$ contra cerca de $29 \%$ de resultados positivos. E com relação aos totais anuais a porcentagem situa por volta dos $79 \%$ para a tendência negativa e próximo de $14 \%$ para a tendência positiva.

Levando-se em consideração os resultados separados da Regressão Linear, do Teste de Mann-Kendall e do Teste do Sinal, a porcentagem das tendências negativas para os meses do ano como um todo são respectivamente $75 \%, 70 \%$ e $54 \%$ e para os totais anuais são de $90 \%, 85 \%$ e $62 \%$, respectivamente. Em contrapartida, 
as porcentagens positivas nos meses do ano em cada um dos testes são na devida ordem $25 \%$, 30\% e $32 \%$ e nos totais anuais são 10\%, 13\% e 19\%, respectivamente.

Outros estudos têm constatado essa diminuição da pluviometria na região estudada. Assis (2016), analisando 36 postos pluviométricos (1964-2014) no trecho submédio da Região Hidrográfica do Rio São Francisco, retrata que, de forma geral, houve tendência de diminuição na pluviometria no período estudado e mostrou que este resultado é mais intenso "na parte Norte, concentrada no estado do Pernambuco e, em menor evidência, no setor baiano do Submédio São Francisco" (p. 102).

Quadro 4. Resultados dos Testes de Tendências das Séries Pluviométricas

\begin{tabular}{|c|c|c|c|c|c|c|c|c|c|c|c|c|c|c|c|c|c|c|c|c|c|c|c|c|c|c|c|c|c|c|c|c|c|c|c|c|c|}
\hline & & & & & & & ז & & INE & & & & & & & & TES & STE & $=$ & MAN & $N N-r$ & KEN & IDAL & & & & & & & & & & & $\mid$ & NAL & & \\
\hline & & & & & & & & & & & & & & & & & & & UB- & $B A C$ & $\overline{C I A C}$ & & & & & & & & & & & & & & & & \\
\hline & & & & & & $\operatorname{ses}$ & do $\mathrm{A}$ & & & & & & \begin{tabular}{|l|l|} 
Total \\
\end{tabular} & & & & & & ses & do $A$ & & & & & & Total & & & & & Mes & $\operatorname{sesc}$ & do $A$ & & & & \\
\hline & $\mathrm{J}$ & $\mathrm{F}$ & \begin{tabular}{|l|}
$\mathrm{M}$ \\
\end{tabular} & $\mathrm{A} \mid$ & $\mathrm{M}$ & $\mathrm{J}$ & $\mathrm{J}$ & A & \begin{tabular}{|c|}
$\mathrm{s}$ \\
\end{tabular} & 0 & $\mathrm{~N}$ & $\mathrm{D}$ & Anual & $\mathrm{J}$ & \begin{tabular}{|l|l|l|}
$F$ &
\end{tabular} & \begin{tabular}{|l|}
$M$ \\
\end{tabular} & $\mathrm{~A}$ & $M$ & $\mathrm{~J}$ & $\mathrm{~J}$ & $\mathrm{~A}$ & $\mathrm{~s}$ & 0 & $\mathrm{~N}$ & $\mathrm{D}$ & Anual & $\mathrm{J}$ & $\mathrm{F}$ & M & $\mathrm{A}$ & M & $\mathrm{J}$ & $\mathrm{J}$ & A & $\mathrm{s}$ & o & $\mathrm{N}$ \\
\hline Total de Positivos & 4 & \begin{tabular}{|c|}
6 \\
\end{tabular} & 31 & 8 & 2 & 13 & 0 & 1 & \begin{tabular}{|l|l|}
3 & $y$ \\
\end{tabular} & 4 & 10 & \begin{tabular}{|l|}
13 \\
\end{tabular} & 6 & 6 & \begin{tabular}{|l|l|}
9 & \\
\end{tabular} & 33 & 12 & 2 & 28 & 2 & 1 & 2 & 3 & 14 & 11 & 7 & 9 & 3 & 23 & 10 & 5 & 24 & 12 & 4 & 10 & 4 & 27 \\
\hline Total c & - & - & - & - & - & - & - & - & - & - & - & - & - & 0 & 0 & 0 & \begin{tabular}{|l|}
1 \\
\end{tabular} & 0 & 1 & 0 & \begin{tabular}{|l|}
0 \\
\end{tabular} & 0 & 0 & 0 & 0 & 0 & 10 & 4 & 4 & 8 & 6 & 3 & 7 & 8 & 11 & 8 & 7 \\
\hline Total de Negativos & 33 & 31 & 6 & 29 & 35 & 24 & 37 & 36 & 34 & 33 & 27 & 24 & 31 & 31 & \begin{tabular}{|l|l|l|l|}
28 & \\
\end{tabular} & 4 & 24 & 35 & 8 & 35 & 36 & 35 & 34 & \begin{tabular}{|l|l}
23 & \\
\end{tabular} & 26 & 30 & 18 & $30 \mid$ & $10 \mid$ & 19 & 26 & 10 & 18 & 25 & 16 & 25 & 3 \\
\hline & & & & & & & & & & & & & & & & & & & UB- & $B A C$ & CIA & & & & & & & & & & & & & & & & \\
\hline & & & & & & ses & do $A$ & & & & & & Total & & & & & & ses & do $A$ & & & & & & Total & & & & & Mes & $\operatorname{sesc}$ & do $A$ & & & & \\
\hline & $\mathrm{J}$ & $F$ & $M$ & A & $M$ & $\mathrm{~J}$ & \begin{tabular}{|l|}
$\mathrm{J}$ \\
\end{tabular} & $\mathrm{A}$ & \begin{tabular}{|l|}
$S$ \\
\end{tabular} & 0 & $\mathrm{~N}$ & $\mathrm{D}$ & Anual & $\mathrm{J}$ & $F$ & \begin{tabular}{|l|l|} 
M \\
\end{tabular} & A & $M$ & \begin{tabular}{|l|}
$\mathrm{J}$ \\
\end{tabular} & \begin{tabular}{|l|}
$\mathrm{J}$ \\
\end{tabular} & \begin{tabular}{|l|}
$A$ \\
\end{tabular} & $S$ & 0 & $\mathrm{~N}$ & $\mathrm{D}$ & Anual & \begin{tabular}{|l}
$J$ \\
\end{tabular} & $F$ & $M$ & A & $M$ & $\mathrm{~J}$ & $\mathrm{~J}$ & \begin{tabular}{|l|}
$A$ \\
\end{tabular} & $S$ & 0 & $\mathrm{~N}$ \\
\hline Total & 0 & 11 & \begin{tabular}{|l|}
19 \\
\end{tabular} & 4 & 0 & 7 & \begin{tabular}{|l|}
0 \\
\end{tabular} & 2 & \begin{tabular}{|l|}
0 \\
\end{tabular} & 9 & 8 & \begin{tabular}{|l|}
16 \\
\end{tabular} & 1 & \begin{tabular}{|l|}
0 \\
\end{tabular} & \begin{tabular}{l|l}
1 \\
\end{tabular} & \begin{tabular}{|l|}
19 \\
\end{tabular} & 10 & 1 & 18 & 0 & \begin{tabular}{|l|}
2 \\
\end{tabular} & \begin{tabular}{|l|} 
\\
\end{tabular} & 5 & 8 & \begin{tabular}{ll|}
16 \\
\end{tabular} & 3 & 5 & 0 & 17 & 6 & 6 & 10 & 2 & \begin{tabular}{|l|}
7 \\
\end{tabular} & 3 & 4 & 12 \\
\hline Total & - & \begin{tabular}{|l|}
- \\
\end{tabular} & - & - & - & - & - & - & - & - & - & \begin{tabular}{|l|}
- \\
\end{tabular} & - & 0 & $\begin{array}{ll}0 \\
\end{array}$ & 0 & \begin{tabular}{|l|l|}
2 \\
\end{tabular} & 0 & \begin{tabular}{|l|}
0 \\
\end{tabular} & 0 & \begin{tabular}{|l|}
0 \\
\end{tabular} & 0 & 1 & 0 & 0 & 1 & 2 & 3 & 5 & 4 & 2 & 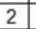 & 0 & 3 & 6 & \begin{tabular}{l|l|}
5 \\
\end{tabular} & 8 \\
\hline otal & 22 & 21 & 3 & \begin{tabular}{|l|}
18 \\
\end{tabular} & 22 & 15 & 22 & 20 & \begin{tabular}{|l|}
22 \\
\end{tabular} & \begin{tabular}{|l|}
13 \\
\end{tabular} & 14 & 6 & 21 & \begin{tabular}{|l|}
22 \\
\end{tabular} & \begin{tabular}{|l|}
21 \\
\end{tabular} & 3 & \begin{tabular}{|l|l|}
10 \\
\end{tabular} & 21 & 4 & \begin{tabular}{|l|}
22 \\
\end{tabular} & 20 & 22 & \begin{tabular}{|l|}
16 \\
\end{tabular} & 14 & 6 & 18 & \begin{tabular}{|l|}
15 \\
\end{tabular} & 19 & 0 & \begin{tabular}{|l|}
12 \\
\end{tabular} & \begin{tabular}{l|l|}
14 & \\
\end{tabular} & 10 & 20 & 12 & $\begin{array}{ll}13 \\
\end{array}$ & 13 & 2 \\
\hline & & & & & & & & & & & & & & & & & & & UB- & $B A C$ & $\mathrm{CIA}$ & & & & & & & & & & & & & & & & \\
\hline & & & & & $\mathrm{Me}$ & ses & do $A$ & & & & & & Total & & & & & & ses & do $\mathrm{A}$ & & & & & & Total & & & & & Mes & sesc & do $\mathrm{A}$ & & & & \\
\hline & $\mathrm{J}$ & \begin{tabular}{|l|} 
\\
\end{tabular} & \begin{tabular}{|l|} 
M \\
\end{tabular} & $A$ & $M$ & $\mathrm{~J}$ & \begin{tabular}{|l|}
$\mathrm{J}$ \\
\end{tabular} & A & \begin{tabular}{|l|}
$\mathrm{S}$ \\
\end{tabular} & 0 & $\mathrm{~N}$ & $\mathrm{D}$ & Anual & \begin{tabular}{|l|}
$\mathrm{J}$ \\
\end{tabular} & \begin{tabular}{|l|l|} 
& \\
\end{tabular} & \begin{tabular}{|l|l|}
$M$ \\
\end{tabular} & A & $M$ & 1 & \begin{tabular}{|l|}
$\mathrm{J}$ \\
\end{tabular} & \begin{tabular}{|l|}
$A$ \\
\end{tabular} & $S$ & 0 & $\mathrm{~N}$ & $\mathrm{D}$ & Anual & \begin{tabular}{|l}
$\mathrm{J}$ \\
\end{tabular} & $\mathrm{F}$ & $M$ & $\mathrm{~A} \mid$ & $M$ & 1 & 1 & A & $S$ & 0 & $\mathrm{~N}$ \\
\hline Total de Positivos & 0 & 1 & 11 & 0 & 0 & 3 & 0 & 1 & 0 & 0 & 4 & 3 & 1 & 0 & 2 & \begin{tabular}{|l|}
12 \\
\end{tabular} & 2 & 0 & 6 & 0 & 1 & 2 & 0 & 5 & 3 & 1 & 2 & 0 & 10 & 2 & 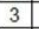 & 4 & 3 & 4 & 3 & 0 & 5 \\
\hline Tot & - & - & - & - & - & - & - & - & - & - & - & - & - & 0 & 0 & 0 & 0 & 0 & 0 & 0 & 0 & 0 & 0 & 0 & 0 & 0 & 1 & 0 & 0 & 1 & 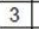 & 1 & & 0 & 1 & 0 & 3 \\
\hline & 12 & 11 & 1 & \begin{tabular}{|l|}
12 \\
\end{tabular} & 12 & 9 & 12 & 11 & - & 12 & 8 & 9 & 11 & 12 & 10 & 0 & 10 & 12 & 6 & 12 & 11 & 10 & \begin{tabular}{|l|l|l|}
12 & \\
\end{tabular} & 7 & 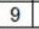 & 11 & 9 & 12 & 2 & 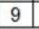 & 6 & & & 8 & 8 & 12 & 4 \\
\hline & & & & & & & & & & & & & & & & & & & UB- & $B A C$ & CIA & & & & & & & & & & & & & & & & \\
\hline & & & & & & $\operatorname{ses}$ & do $A$ & & & & & & Total & & & & & & ses & do $A$ & & & & & & \begin{tabular}{|l|} 
Total \\
\end{tabular} & & & & & Mes & $\operatorname{ses}$ & do $A$ & & & & \\
\hline & $\mathrm{J}$ & $\mathrm{F}$ & \begin{tabular}{|l|}
$\mathrm{M}$ \\
\end{tabular} & $m$ & $\mathrm{M}$ & $\mathrm{J}$ & $\mathrm{J}$ & A & \begin{tabular}{|c|}
$\mathrm{S}$ \\
\end{tabular} & 0 & $\mathrm{~N}$ & $\mathrm{D}$ & Anual & $\mathrm{J}$ & $F$ & \begin{tabular}{|l|}
$\mathrm{M}$ \\
\end{tabular} & A & $M$ & $\mathrm{~J}$ & $\mathrm{~J}$ & $\mathrm{~A}$ & $\mathrm{~s}$ & 0 & $\mathrm{~N}$ & $\mathrm{D}$ & Anual & \begin{tabular}{|l|l|l} 
\\
\end{tabular} & & $M$ & & M & & 1 & $A$ & $\mathrm{~s}$ & $\mathrm{O}$ & $\mathrm{N}$ \\
\hline Tota & 0 & 1 & 6 & 3 & 1 & 1 & 0 & 1 & \begin{tabular}{|l|}
1 \\
\end{tabular} & 0 & 3 & \begin{tabular}{|l|}
2 \\
\end{tabular} & 0 & 0 & 1 & 6 & 4 & 1 & 3 & 0 & 0 & 1 & 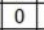 & 4 & 4 & 0 & , & 0 & & & & & & & 2 & 0 & 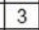 \\
\hline To & - & 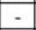 & - & - & - & - & - & - & - & - & - & . & - & \begin{tabular}{|l|l}
0 \\
\end{tabular} & 0 & 0 & 0 & $\begin{array}{l}0 \\
\end{array}$ & 0 & 0 & \begin{tabular}{|l|}
0 \\
\end{tabular} & 0 & 0 & 0 & & 7 & 0 & 0 & 1 & 3 & 1 & & & 0 & 0 & 0 & 1 \\
\hline Tota & 6 & 5 & 0 & 3 & 5 & 5 & \begin{tabular}{|l|}
6 \\
\end{tabular} & 5 & \begin{tabular}{|l|}
5 \\
\end{tabular} & 6 & 3 & 4 & 6 & 6 & 5 & 0 & 2 & 5 & 3 & 6 & 6 & 5 & 6 & 2 & 2 & 5 & 6 & 6 & 0 & 2 & 3 & 3 & 6 & 6 & 4 & 6 & 2 \\
\hline & & & & & & & & & & & & & & & & & & & UB- & $B A C$ & CIA & & & & & & & & & & & & & & & & \\
\hline & & & & & & ses & do $A$ & & & & & & Total & & & & & & ses & do $A$ & & & & & & Total & & & & & Mes & $\operatorname{ses}$ & do $A$ & & & & \\
\hline & $\mathrm{J}$ & \begin{tabular}{|l|}
$F$ \\
\end{tabular} & \begin{tabular}{|l|} 
M \\
\end{tabular} & A & $M$ & $\mathrm{~J}$ & \begin{tabular}{|l|}
1 \\
\end{tabular} & $\mathrm{~A}$ & \begin{tabular}{|l|}
$S$ \\
\end{tabular} & 0 & $\mathrm{~N}$ & D & Anual & $\mathrm{J}$ & $F$ & $M$ & A & $M$ & J & $\mathrm{J}$ & $\mathrm{A}$ & S & 0 & $\mathrm{~N}$ & $\mathrm{D}$ & Anual & $\mathrm{J}$ & $F$ & $M$ & \begin{tabular}{|l|l|} 
A & \\
\end{tabular} & $M$ & 1 & $\mathrm{~J}$ & $A$ & S & 0 & $\mathrm{~N}$ \\
\hline Tot & 0 & \begin{tabular}{|l|}
0 \\
\end{tabular} & 5 & 1 & 0 & 0 & 0 & 0 & 1 & 0 & 4 & 0 & 0 & 1 & 0 & 5 & 1 & 2 & 1 & 0 & 0 & 0 & 0 & 4 & 2 & 0 & 0 & 0 & 4 & & 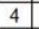 & & & 0 & 3 & 0 & 5 \\
\hline To & - & - & - & - & - & - & 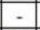 & - & - & - & - & - & & 0 & 0 & 0 & 0 & 0 & 0 & 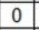 & 0 & 0 & 0 & 0 & & & 0 & 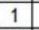 & & & 1 & & & 0 & 0 & 0 & 0 \\
\hline & 5 & \begin{tabular}{|l|}
5 \\
\end{tabular} & 0 & 4 & 5 & 5 & 5 & 5 & 4 & 5 & 1 & 5 & 5 & 4 & 5 & 0 & 4 & 3 & 4 & 5 & 5 & 5 & 5 & 1 & 3 & & 5 & 4 & 0 & 3 & 0 & 3 & & 5 & 2 & 5 & 0 \\
\hline & & & & & & & & & & & & & & & & & & & UB. & $B_{1}$ & CIA & & & & & & & & & & & & & & & & \\
\hline & & & & & & $s \in$ & 00 & & & & & & Tote & & & & & & ses & do $A$ & & & & & & Total & & & & & Mes & ; & do $A$ & & & & \\
\hline & $\mathrm{J}$ & $\mathrm{F}$ & \begin{tabular}{|l|} 
M \\
\end{tabular} & A & $M$ & $\mathrm{~J}$ & $\mathrm{~J}$ & A & $\mathrm{S}$ & 0 & $\mathrm{~N}$ & $D$ & Anual & $\mathrm{J}$ & & $M$ & A & $M$ & & $\mathrm{~J}$ & $A$ & 3 & 0 & $\mathrm{~N}$ & $\mathrm{D}$ & Anual & $\mathrm{J}$ & & & & $M$ & & $\mathrm{~J}$ & A & $S$ & 0 & $N$ \\
\hline Tote & 0 & \begin{tabular}{|l|}
0 \\
\end{tabular} & 8 & 7 & 4 & 0 & 0 & 1 & 1 & 0 & 9 & 1 & 0 & 0 & 0 & 80 & 7 & 3 & 1 & 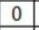 & 0 & 1 & 80 & 9 & 1 & 0 & 0 & 0 & & 3 & 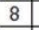 & & & 0 & 6 & 2 & 8 \\
\hline & - & - & - & - & - & - & - & - & - & - & - & - & - & 0 & 0 & 0 & 0 & 0 & 0 & 0 & 0 & 0 & 0 & 0 & 0 & 0 & 0 & 2 & & & c & & 0 & 0 & 0 & ) & 2 \\
\hline Tot & \begin{tabular}{|l|}
10 \\
\end{tabular} & 10 & 2 & 3 & 6 & 10 & 10 & 9 & 9 & 10 & 1 & 9 & 10 & \begin{tabular}{|l|l|}
10 \\
\end{tabular} & \begin{tabular}{|l|}
10 \\
\end{tabular} & 2 & 3 & 1 & 9 & \begin{tabular}{|l|l|}
10 \\
\end{tabular} & 10 & \begin{tabular}{|l|}
9 \\
\end{tabular} & \begin{tabular}{|l|l|}
10 \\
\end{tabular} & 1 & 9 & 10 & 10 & 8 & 1 & 4 & 2 & 9 & 10 & 10 & 4 & 8 & 0 \\
\hline & & & & & & & & & & & & & & & & & & & UB & $B A C$ & $C I A$ & & & & & & & & & & & & & & & & \\
\hline & & & & & Mes & ses & do $A$ & & & & & & Tota & & & & & & ses & do $A$ & & & & & & \begin{tabular}{|l|} 
Total \\
\end{tabular} & & & & & Mes & $\operatorname{ses}$ & do $A$ & & & & \\
\hline & $\mathrm{J}$ & \begin{tabular}{|l|}
$F$ \\
\end{tabular} & $M$ & A & $M$ & $\mathrm{~J}$ & \begin{tabular}{|l|}
$\mathrm{J}$ \\
\end{tabular} & A & $\begin{array}{ll} \\
\end{array}$ & 0 & $\mathrm{~N}$ & D & $\mathrm{Ar}$ & $\mathrm{J}$ & $F$ & M & A & $M$ & 4 & J & A & $S$ & 0 & N & $\mathrm{D}$ & Anual & \begin{tabular}{|l}
$J$ \\
\end{tabular} & & & A & 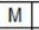 & & 1 & A & $S$ & 01 & N \\
\hline & 0 & 0 & 5 & 9 & 12 & 5 & 1 & 2 & \begin{tabular}{|l|}
1 \\
\end{tabular} & 5 & 11 & 3 & 2 & 0 & 2 & . & 7 & 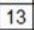 & & 2 & 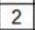 & 0 & 0 & 10 & & 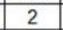 & 0 & 3 & & & 10 & & & 2 & 1 & \begin{tabular}{|l|}
0 \\
\end{tabular} & 14 \\
\hline Tot & - & - & - & - & - & - & - & - & $\begin{array}{ll}- \\
\end{array}$ & - & - & - & - & 0 & 0 & 0 & , & 0 & 1 & 0 & \begin{tabular}{|l|}
0 \\
\end{tabular} & 0 & 1 & 0 & 0 & & 2 & 5 & & 2 & 3 & 3 & 2 & 1 & 0 & 5 & 0 \\
\hline Total & 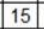 & 15 & \begin{tabular}{|l|l|}
10 \\
\end{tabular} & 6 & 3 & 10 & 14 & 13 & \begin{tabular}{|l|l|}
14 \\
\end{tabular} & \begin{tabular}{|l|}
10 \\
\end{tabular} & 4 & \begin{tabular}{|l|}
12 \\
\end{tabular} & 1. & \begin{tabular}{|l|}
15 \\
\end{tabular} & 13 & 7 & 8 & 2 & 13 & \begin{tabular}{|l|}
13 \\
\end{tabular} & \begin{tabular}{|l|}
13 \\
\end{tabular} & 15 & \begin{tabular}{|l|}
14 \\
\end{tabular} & 5 & \begin{tabular}{|l|}
11 \\
\end{tabular} & 13 & \begin{tabular}{|l|l|}
13 & \\
\end{tabular} & 7 & 4 & 6 & 2 & 8 & 12 & 12 & 14 & 10 & 1 \\
\hline & & & & & & & & & & & & & & & & & & & & 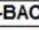 & & & & & & & & & & & & & & & & & \\
\hline & & & & & Mes & ses & do $A$ & & & & & & \begin{tabular}{|l|l|} 
Total \\
\end{tabular} & & & & & & ses & do $A$ & & & & & & Total & & & & & Mes & ses & do $A$ & & & & \\
\hline & $\mathrm{J}$ & \begin{tabular}{|l|}
$F$ \\
\end{tabular} & $M$ & $A$ & $M$ & $\mathrm{~J}$ & \begin{tabular}{|l}
$J$ \\
\end{tabular} & A & \begin{tabular}{|l|}
$\mathrm{S}$ \\
\end{tabular} & 0 & $\mathrm{~N}$ & $\mathrm{D}$ & Anual & $\mathrm{J}$ & & $M$ & $A$ & $M$ & , & 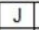 & $\mathrm{A}$ & $S$ & 0 & $\mathrm{~N}$ & $\mathrm{D}$ & Anual & \begin{tabular}{|l}
$\mathrm{J}$ \\
\end{tabular} & $F_{-}$ & M & & $M$ & $T$ & 1 & $A$ & S & 0 & $\mathrm{~N}$ \\
\hline & 2 & \begin{tabular}{|l|}
0 \\
\end{tabular} & $\begin{array}{l} \\
\end{array}$ & 1 & 1 & 0 & 2 & 1 & \begin{tabular}{|l|l|}
1 \\
\end{tabular} & 2 & 1 & 0 & 0 & 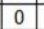 & 0 & 0 & 1 & 3 & 2 & $=$ & 2 & 1 & 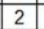 & 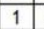 & 0 & 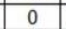 & $=$ & 0 & & & & & & 2 & 1 & ) & \\
\hline & - & - & 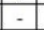 & - & - & - & - & 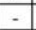 & - & - & 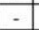 & - & & 0 & 0 & 0 & 1 & 0 & 0 & 0 & 0 & 0 & 0 & 0 & & & 0 & 0 & 0 & 0 & 1 & 1 & & 0 & 1 & 1 & 1 \\
\hline Tota & 1 & 3 & 3 & 2 & 2 & 3 & 1 & 2 & 2 & 1 & 2 & 3 & 3 & 3 & 3 & 3 & 1 & 0 & 1 & 1 & \begin{tabular}{|l|}
1 \\
\end{tabular} & 2 & 1 & 2 & 3 & & 3 & 3 & 2 & 2 & 0 & 0 & & 1 & 1 & 2 & 1 \\
\hline & & & & & & & & & & & & & & & & & & & $\mathrm{UB}$ - & - & & & & & & & & & & & & & & & & & \\
\hline & & & & & & se & 00 & & & & & & Total & & & & & & ses & do $A$ & & & & & & \begin{tabular}{|l|} 
Total \\
\end{tabular} & & & & & Mes & $\mathrm{se}$ & d & & & & \\
\hline & $\mathrm{J}$ & $F$ & M & A & M & $\mathrm{J}$ & $\mathrm{J}$ & A & \begin{tabular}{|l|}
$S$ \\
\end{tabular} & 0 & $\mathrm{~N}$ & $D$ & Anual & $\mathrm{J}$ & $F$ & M & A & $M$ & 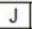 & $\mathrm{J}$ & A & S & 0 & $\mathrm{~N}$ & $\mathrm{D}$ & Anual & \begin{tabular}{|l}
$J$ \\
\end{tabular} & $F$ & $M$ & $\mathrm{~A} \mid$ & $M$ & $\mathrm{~J}$ & $\mathrm{~J}$ & A & $S$ & 0 & $\mathrm{~N}$ \\
\hline & 4 & \begin{tabular}{|l|}
3 \\
\end{tabular} & 0 & 3 & 4 & 1 & 5 & 6 & \begin{tabular}{|l|}
1 \\
\end{tabular} & 8 & 2 & 0 & 1 & 3 & 6 & 0 & 4 & 8 & 4 & 1 & 8 & 2 & 4 & 1 & 0 & 1 & 3 & 6 & 1 & 0 & 7 & 3 & 4 & 8 & 1 & 6 & 3 \\
\hline & - & - & - & - & - & - & - & - & - & - & - & - & - & 0 & 0 & 0 & 0 & 0 & 1 & 0 & 0 & 0 & 0 & 0 & 0 & & 1 & 1 & 1 & 2 & 1 & 0 & 2 & 0 & 1 & 1 & 1 \\
\hline & 6 & \begin{tabular}{|l|}
7 \\
\end{tabular} & \begin{tabular}{|l|}
10 \\
\end{tabular} & 7 & 6 & 9 & 5 & 4 & 9 & 2 & 8 & 10 & 9 & 7 & 4 & \begin{tabular}{|l|l|}
10 \\
\end{tabular} & 6 & 2 & 5 & 3 & 2 & 8 & 6 & 9 & 10 & 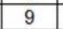 & 6 & 3 & 8 & 8 & 2 & 7 & 4 & 2 & 8 & 3 & 6 \\
\hline & & & & & & & & & & & & & & & & & & & & $B A$ & CIA & & & & & & & & & & & & & & & & \\
\hline & & & & & & $\mathrm{se}$ & do $\mathrm{A}$ & & & & & & Total & & & & & & ses & do $A$ & & & & & & \begin{tabular}{|l|} 
Total \\
\end{tabular} & & & & & Mes & ses & do $\mathrm{A}$ & & & & \\
\hline & J & $\mathrm{F}$ & M & $A$ & $M$ & $\mathrm{~J}$ & $\mathrm{~J}$ & A & & 0 & $\mathrm{~N}$ & $D$ & Anua & 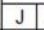 & & $M$ & $m$ & $M$ & & $\mathrm{~J}$ & $\mathrm{~A}$ & $\mathrm{~S}$ & 0 & $\mathrm{~N}$ & 5 & Anual & $\mathrm{J}$ & & M & & $M$ & $\mathrm{~J}$ & & $A$ & $\mathrm{~s}$ & 0 & $\mathrm{~N}$ \\
\hline Tota & 2 & 1 & 0 & 0 & 3 & 3 & 3 & 4 & 1 & 4 & 2 & 1 & 1 & 2 & 3 & 0 & 1 & 4 & 4 & 4 & 4 & 2 & 4 & 0 & & 2 & 1 & 1 & 0 & 0 & 4 & 1 & 3 & 4 & 2 & 4 & 1 \\
\hline & - & - & - & - & - & 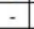 & - & 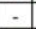 & - & - & - & - & - & 0 & 0 & 0 & 0 & 0 & 0 & 0 & 0 & 0 & 0 & 0 & 0 & 0 & 0 & 2 & 0 & 1 & 0 & 3 & 0 & 0 & 1 & 0 & 0 \\
\hline otal de Negativos & 2 & 3 & 4 & 4 & 1 & 1 & 1 & 0 & 3 & 0 & 2 & 3 & 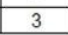 & 2 & 1 & 4 & 3 & 0 & 0 & 0 & 0 & 2 & 0 & 4 & 4 & 2 & 3 & & 4 & 3 & 0 & 0 & 1 & \begin{tabular}{|l|}
0 \\
\end{tabular} & 1 & 0 & 3 \\
\hline
\end{tabular}

Fonte: Organizado pelos autores

Silva, Souza e Azevedo (2012) avaliaram índices de detecção de mudanças climáticas no estado da Bahia no período de 1970 a 2006 e chegaram à conclusão de que a região do Baixo Médio São Francisco apresentou redução das chuvas diárias e do total da precipitação anual e aumento do número de dias com temperatura máxima diária. 
Soares et al. (2018), num estudo sobre indicadores climáticos de desertificação na bacia hidrográfica do rio Pajéu (PE), constataram que dos 12 postos pluviométricos analisados (de 1965 a 2014) dez apresentaram tendência negativa, mostrando que a altura anual de chuvas está diminuindo e que "a redução dos valores do Índice de Aridez (IA) podem indicar uma mudança da categoria do semiárido (IA entre 0,20 e $0,50)$ para árido $(0,05$ a 0,20$) "$ (p. 371).

Projeções apresentadas por Marengo et al. (2007) para o Nordeste brasileiro indicam que praticamente todos os modelos utilizados convergiram numa situação de clima mais seco.

Conforme estudo de Silveira et al. (2016), utilizando as projeções de precipitação e temperatura dos modelos globais do CMIP5 (Couple Model Intercomparison Project Phase 5), na Região Hidrográfica do Rio São Francisco "todos os modelos apresentaram tendência positiva para a temperatura no período de 2011 a 2100 e divergem quanto ao futuro das precipitações. Apesar dessa divergência, o conjunto de modelos projetam anomalias entre $-20 \%$ e $20 \%$ em cada período de 30 anos" (p. 426) na variável chuva. E concluem dizendo que as divergências dos modelos analisados corroboram com o alto nível de incertezas nas projeções do CMIP5, mas que mesmo assim as mesmas ajudam a definir possíveis cenários futuros de precipitação e temperatura, que podem ser utilizados nas políticas e gestão da Região Hidrográfica do Rio São Francisco.

Mais especificamente com relação aos resultados sobre o total anual é plausível afirmar que as sub-bacias 43, 44, 45 e 47 não tiveram nenhuma ocorrência de tendência positiva em nenhum dos métodos utilizados na pesquisa aqui relatada. Ademais, em exceção às sub-bacias 48 e 49 , em todas as outras sub-bacias prevalece a tendência negativa em todos os testes. Na sub-bacia 48 o teste do sinal teve a mesma quantidade de ocorrências positivas e negativas e quatro ocorrências ditas "normais". E na sub-bacia 49, houve também o mesmo total de ocorrências positivas e negativas no teste de Mann-Kendall e no teste do sinal prevalece a tendência positiva em relação à negativa.

Com relação ao total de ocorrências mensais, na Regressão Linear a maioria das sub-bacias possuem uma porcentagem de ocorrência de tendência negativa acima de $70 \%$, exceto nas sub-bacias 47, 48 e 49 . Destaca-se que as sub-bacias 42 e 44 têm respectivamente, $84 \%$ e $82 \%$ de eventos negativos. Há uma maior tendência de diminuição da pluviometria no alto e médio São Francisco, se comparado com o sub-médio e baixo São Francisco. Nas tendências positivas deste teste a porcentagem está entre $16 \%$ e $31 \%$, a não ser na sub-bacia 49 que teve $50 \%$.

Já no teste de Mann-Kendall todas as sub-bacias apresentam uma porcentagem de ocorrências mensais com tendência negativa entre $58 \%$ a $77 \%$, dando destaque também às porções do alto e médio São Francisco. E nas tendências positivas a porcentagem está em torno de $23 \%$ a $39 \%$, à exceção da sub-bacia 49 que tem $58 \%$. Neste teste vale ressaltar que a classe "normal" (que a tendência é de manutenção) não teve resultados expressivos, ou seja, a porcentagem ficou em torno de 0 a $3 \%$.

No Teste do Sinal os resultados se mostram menos discrepantes entre as classes positiva, normal e negativa que em relação aos outros dois métodos. A porcentagem do total de tendência negativa gira em torno de $40 \%$ a $64 \%$, na tendência positiva é entre $25 \%$ a $46 \%$ e a classe normal ficou em torno de $8 \%$ a $19 \%$.

Tal como abordado anteriormente, há uma diminuição das chuvas na Região Hidrográfica do Rio São Francisco no intervalo deste estudo (1976-2015). Pode-se afirmar que dos 12 meses do ano, em 11 meses foram constatadas a predominância 
no decréscimo da pluviometria na Regressão Linear e 10 meses em cada um dos outros dois testes.

Os meses com significativas ocorrências de diminuição da pluviometria na região hidrográfica nos três testes são Janeiro, Fevereiro, Julho e Setembro, além do total anual. Em contrapartida, os meses com expressivas ocorrências de aumento pluviométrico nas três ferramentas são Março e Novembro.

De modo geral, os resultados por mês nos três testes são relativamente similares. Nesta lógica, destaca-se que os produtos finais com maior similaridade nos resultados entre as ferramentas estatísticas utilizadas são Janeiro, Fevereiro, Março, Julho, Agosto e Dezembro e os resultados mais discrepantes entre os métodos ocorrem nos meses de Maio, Junho e Novembro. Em específico ao mês de Julho os resultados dos três testes deram iguais, salvo na sub-bacia 48, que possui a mesma quantidade de ocorrências positivas e negativas no Teste do Sinal.

Apesar do grande desafio matemático e computacional de se propor modelos e/ou métodos que realmente possam identificar tendências climáticas, e também levando em consideração a incerteza da ação humana futura, as três ferramentas estatísticas utilizadas nesta pesquisa têm fácil manuseio e se mostraram bastante coerentes entre si. Embora os três testes aqui aplicados sejam amplamente utilizados em estudos de tendências climáticas, as análises feitas neste artigo representam apenas possibilidades de eventuais mudanças no clima. Se considerarmos que a tendência de diminuição da pluviometria na área de estudo é um fato, deve-se estudar cada vez mais suas repercussões locais para minimizar suas consequências.

\section{AVALIAÇÃO DOS EVENTOS DE EXTREMOS PLUVIOMÉTRICOS}

A frequência de extremos pluviométricos em termos de totais anuais pode ser analisada por meio dos produtos do SPI e do RAI nas Figuras 3 e 4, respectivamente. Percebe-se, em relação ao SPI, que em todas as sub-bacias a porcentagem de ocorrência de eventos ditos normais (N) é acima de $40 \%$ e que as porcentagens de episódios de anomalias mais extremas variam entre 1,3 a 2,5\% nos extremamente úmidos e 0,3 a 2,3\% nos extremamente secos. A porcentagem das outras classes muito úmido (A2), moderadamente úmido (A1), muito seco (V2) e moderadamente seco (V1), em grande parte das sub-bacias têm mais que $10 \%$, dando destaque as sub-bacias que possuem mais que $15 \%$ de ocorrências, mais presentes nas classes A2 e V1.

Em relação aos resultados do RAl é presumível verificar que as classes úmido (C1) e seco (S1) possuem as porcentagens mais significativas que se comparado as outras classes em todas as sub-bacias, estando em grande maioria acima de $28 \%$. Pode ser observado também que a medida que percorre-se as classes até os extremamente úmidos (C3) e secos (S3) a porcentagem de ocorrências vai diminuindo. 
Figura 3. Porcentagem (\%) do $\mathrm{n}^{\circ}$ de ocorrências nos totais anuais por classes do SPI por Sub-bacia

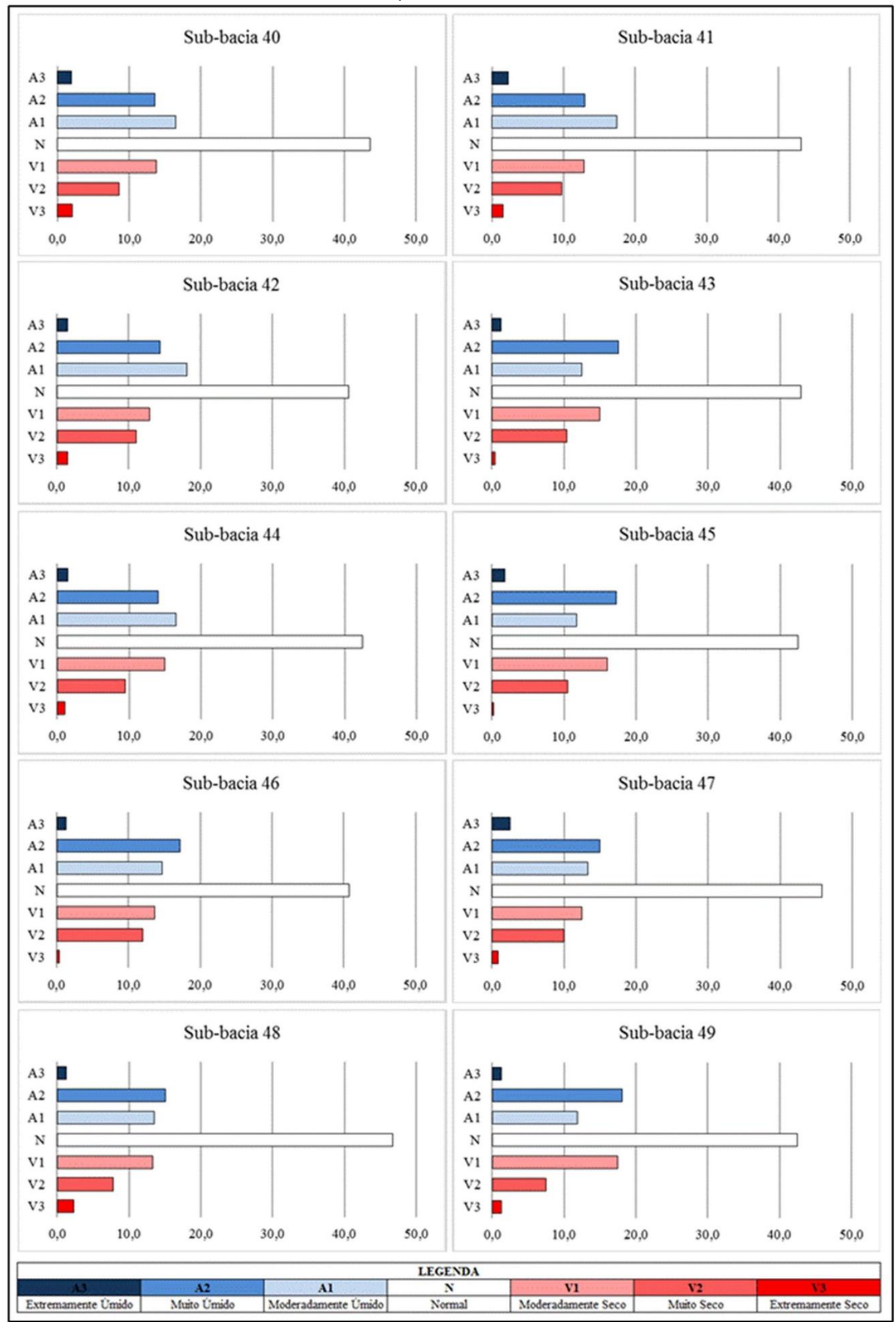

Fonte: Organizado pelos autores 
Figura 4. Porcentagem (\%) do $\mathrm{n}^{\circ}$ de ocorrências nos totais anuais por classes do RAI por Sub-bacia

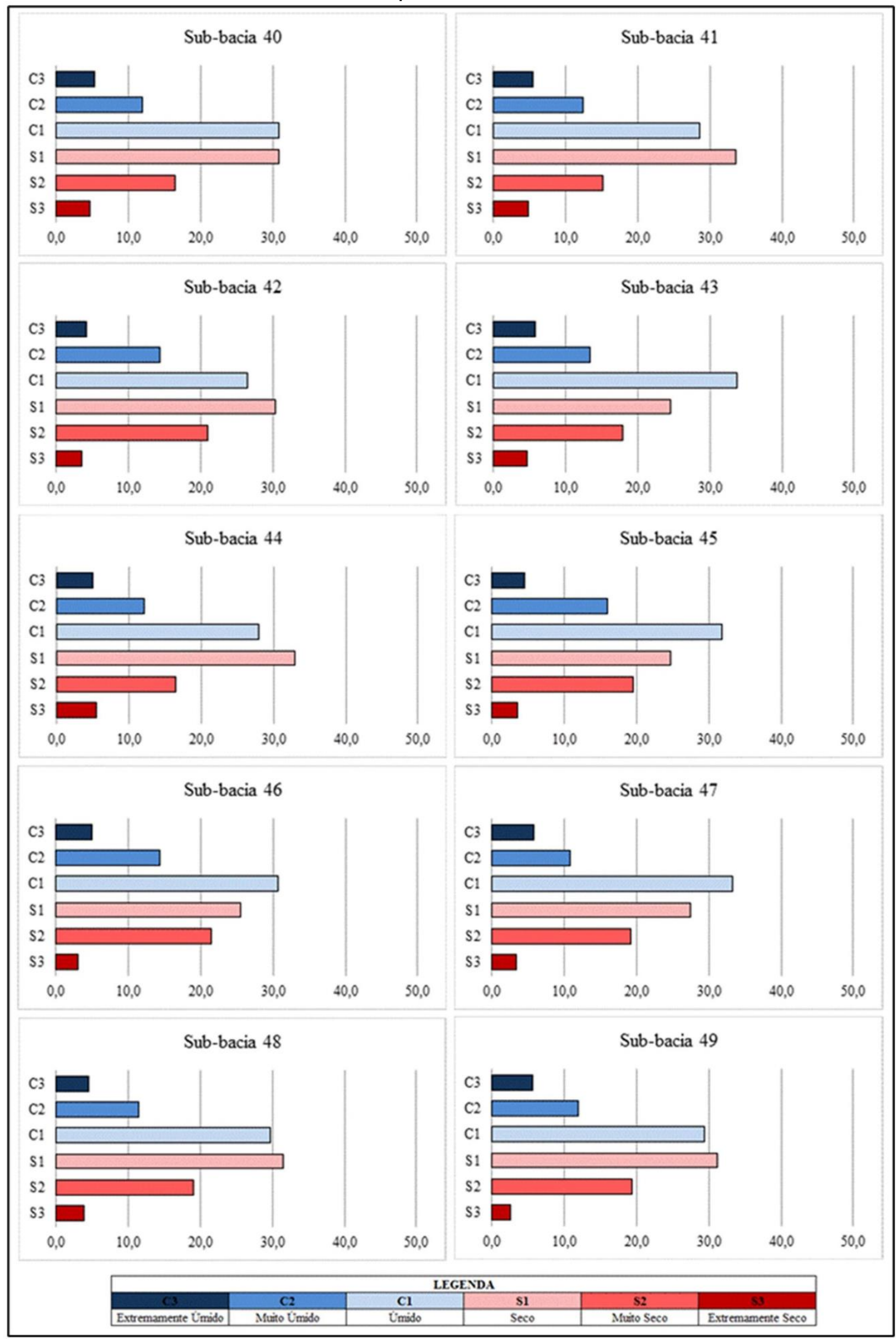

Fonte: Organizado pelos autores 
Comparando os resultados das classes extremas dos métodos SPI e RAI notase que a porcentagem dos extremos úmidos e secos é maior no RAI que no SPI. Mas, ambas as ferramentas matemáticas demonstram que os eventos expressivos estão relativamente distribuídos na série temporal utilizada (1976-2015). E também é verificado que a ocorrência de anos normais é mais expressiva no SPI do que no RAI.

Em relação às anomalias positivas e negativas mensais, também observadas através dos resultados do SPI e do RAI (Quadros 5 e 6, respectivamente), pode-se evidenciar quanto aos produtos do SPI que $45 \%$ do total de ocorrências mensais são normais e que a diferença de porcentagem do somatório dos episódios úmidos e secos é de apenas $5 \%$ e que há mais eventos nas classes subsequentes da classe normal e diminui bastante nas classes extremamente úmido (A3) e extremamente $\operatorname{seco}(\mathrm{V} 3)$.

Ao analisar o SPI por sub-bacia nota-se que o padrão observado para a região hidrográfica é o mesmo para praticamente todas as sub-bacias, exceto na $44,45 \mathrm{e}$ 46. Nestas pode-se averiguar que a porcentagem de ocorrências na classe normal (N) é menor e que na classe moderadamente úmido (A1) é maior.

Nos meses de Maio a Setembro a porcentagem de ocorrência de eventos na classe extremamente seco (V3) é praticamente nula. Já nos meses de Junho e Agosto são os que apresentam as maiores porcentagens de episódios, sendo estes localizados na classe normal (N). Além disso, pode-se observar que os meses de Junho, Julho e Agosto apontam uma menor porcentagem de episódios nas classes V1 e V2 em relação aos outros meses e uma maior porcentagem de eventos nas classes A1 e A2.

Já nos resultados do RAI, pode-se verificar que a classe muito seco (S2) é a que apresenta a maior porcentagem de ocorrências dentro da região hidrográfica (37\%) e também isso é válido para cada sub-bacia individualmente. Vale ressaltar ainda que, em especial na sub-bacia 47 essa classe apresenta mais que $49 \%$ do total de eventos. Devido a esse fato a diferença entre a porcentagem de episódios úmidos e secos é de $-24 \%$.

Além do mais, nessa mesma classe (S2), os meses de Junho, Julho e Agosto são os que apresentam as maiores porcentagens de ocorrências mensais com, respectivamente, $59 \%, 63 \%$ e $61 \%$.

Nas classes mais extremas (C3 - extremamente úmido e S3 - extremamente seco), é presumível observar que $\mathrm{C} 3$ apresenta $6 \%$ do número de eventos na área de estudo e S3 apenas 1\%. Esse resultado é verificado também quando se analisa cada sub-bacia, dando destaque à maior diferença entre essas classes na sub-bacia 47 , com $6,7 \%$ de margem positiva.

E se verificar essas mesmas classes nos resultados das porcentagens mensais é possível dizer que os meses de Junho e Julho têm as maiores porcentagens em C3, com 7\% e já em S3 esses meses, juntamente com Maio, Agosto e Setembro exibem porcentagem nula. 


\section{Quadro 5. Número de Ocorrências Mensais por Classes do SPI por Sub-bacia}

\begin{tabular}{|c|c|c|c|c|c|c|c|c|c|c|c|c|c|}
\hline \multicolumn{14}{|c|}{ SUB-BACIA 40} \\
\hline Classes do SPI & JAN & FEV & MAR & $\mathrm{ABR}$ & MAI & JUN & JUL & AGO & SET & OUT & NOV & DEZ & $\sum$ DOS MESES \\
\hline $\mathrm{A} 3$ & 3 & 6 & 9 & 7 & 6 & 16 & 12 & 8 & 12 & 8 & 18 & 10 & 115 \\
\hline A2 & 181 & 146 & 150 & 147 & 169 & 186 & 203 & 203 & 130 & 152 & 158 & 159 & 1984 \\
\hline A 1 & 243 & 278 & 247 & 241 & 214 & 138 & 166 & 208 & 271 & 257 & 201 & 228 & 2692 \\
\hline $\mathrm{N}$ & 560 & 567 & 612 & 603 & 620 & 715 & 853 & 823 & 621 & 581 & 623 & 625 & 7803 \\
\hline V1 & 219 & 221 & 207 & 236 & 223 & 393 & 246 & 225 & 169 & 216 & 217 & 217 & 2789 \\
\hline V2 & 251 & 237 & 227 & 221 & 227 & 32 & 0 & 13 & 268 & 247 & 244 & 210 & 2177 \\
\hline V3 & 23 & 25 & 28 & 25 & 21 & 0 & 0 & 0 & 8 & 19 & 19 & 31 & 199 \\
\hline \multicolumn{14}{|c|}{ SUB-BACIA 41} \\
\hline Classes do SPI & JAN & FEV & MAR & $\mathrm{ABR}$ & MAI & JUN & JUL & AGO & SET & OUT & NOV & DEZ & $\sum$ DOS MESES \\
\hline $\mathrm{A} 3$ & 2 & 6 & 3 & 7 & 7 & 17 & 6 & 5 & 6 & 9 & 6 & 12 & 86 \\
\hline A2 & 101 & 73 & 95 & 92 & 98 & 92 & 120 & 120 & 94 & 84 & 92 & 83 & 1144 \\
\hline A1 & 133 & 157 & 132 & 135 & 133 & 100 & 130 & 116 & 170 & 142 & 135 & 121 & 1604 \\
\hline $\mathrm{N}$ & 359 & 373 & 375 & 356 & 354 & 571 & 555 & 507 & 309 & 366 & 363 & 387 & 4875 \\
\hline V1 & 132 & 113 & 128 & 147 & 158 & 100 & 63 & 132 & 160 & 139 & 141 & 143 & 1556 \\
\hline V2 & 139 & 142 & 127 & 135 & 129 & 0 & 6 & 0 & 140 & 127 & 125 & 117 & 1187 \\
\hline V3 & 14 & 16 & 20 & 8 & 1 & 0 & 0 & 0 & 1 & 13 & 18 & 17 & 108 \\
\hline \multicolumn{14}{|c|}{ SUB-BACIA 42} \\
\hline Classes do SPI & JAN & FEV & MAR & $\mathrm{ABR}$ & MAI & JUN & JUL & AGO & SET & OUT & NOV & DEZ & DOS MESES \\
\hline A3 & 2 & 4 & 1 & 3 & 5 & 7 & 8 & 6 & 8 & 2 & 3 & 4 & 53 \\
\hline A2 & 50 & 52 & 42 & 50 & 58 & 55 & 43 & 50 & 50 & 52 & 59 & 52 & 613 \\
\hline A1 & 89 & 79 & 90 & 77 & 68 & 50 & 103 & 61 & 71 & 83 & 69 & 74 & 914 \\
\hline $\mathrm{N}$ & 186 & 186 & 212 & 199 & 186 & 368 & 326 & 363 & 190 & 185 & 189 & 199 & 2789 \\
\hline $\mathrm{V} 1$ & 56 & 73 & 52 & 63 & 156 & 0 & 0 & 0 & 123 & 71 & 84 & 69 & 747 \\
\hline V2 & 89 & 79 & 72 & 84 & 7 & 0 & 0 & 0 & 38 & 82 & 70 & 72 & 593 \\
\hline $\mathrm{V} 3$ & 8 & 7 & 11 & 4 & 0 & 0 & 0 & 0 & 0 & 5 & 6 & 10 & 51 \\
\hline \multicolumn{14}{|c|}{ SUB-BACIA 43} \\
\hline Classes do SPI & JAN & FEV & MAR & $\mathrm{ABR}$ & MAI & JUN & JUL & $\mathrm{AGO}$ & SET & OUT & NOV & DEZ & DOS MESES \\
\hline $\mathrm{A} 3$ & 2 & 2 & 0 & 0 & 5 & 2 & 6 & 4 & 5 & 3 & 0 & 3 & 32 \\
\hline A2 & 24 & 30 & 24 & 30 & 22 & 29 & 20 & 24 & 19 & 22 & 15 & 25 & 284 \\
\hline $\mathrm{A} 1$ & 38 & 36 & 39 & 39 & 31 & 30 & 128 & 31 & 40 & 39 & 58 & 32 & 541 \\
\hline $\mathrm{N}$ & 97 & 89 & 106 & 89 & 126 & 179 & 86 & 181 & 92 & 100 & 103 & 104 & 1352 \\
\hline $\mathrm{V} 1$ & 31 & 43 & 32 & 40 & 49 & 0 & 0 & 0 & 84 & 35 & 28 & 41 & 383 \\
\hline $\mathrm{V} 2$ & 44 & 38 & 35 & 40 & 7 & 0 & 0 & 0 & 0 & 40 & 30 & 30 & 264 \\
\hline V3 & 4 & 2 & 4 & 2 & 0 & 0 & 0 & 0 & 0 & 1 & 6 & 5 & 24 \\
\hline \multicolumn{14}{|c|}{ SUB-BACIA 44} \\
\hline Classes do SPI & JAN & FEV & MAR & $\mathrm{ABR}$ & MAI & JUN & JUL & $\mathrm{AGO}$ & SET & OUT & NOV & DEZ & DOS MESES \\
\hline $\mathrm{A} 3$ & 1 & 2 & 0 & 0 & 3 & 3 & 3 & 4 & 1 & 1 & 0 & 5 & 23 \\
\hline $\mathrm{A} 2$ & 22 & 28 & 20 & 25 & 25 & 20 & 18 & 18 & 26 & 18 & 17 & 20 & 257 \\
\hline $\mathrm{A} 1$ & 29 & 22 & 36 & 35 & 21 & 118 & 150 & 91 & 19 & 42 & 38 & 24 & 625 \\
\hline $\mathrm{N}$ & 81 & 83 & 88 & 68 & 124 & 59 & 29 & 87 & 127 & 74 & 87 & 87 & 994 \\
\hline $\mathrm{V} 1$ & 30 & 36 & 17 & 34 & 27 & 0 & 0 & 0 & 27 & 28 & 30 & 36 & 265 \\
\hline $\mathrm{V} 2$ & 36 & 27 & 37 & 37 & 0 & 0 & 0 & 0 & 0 & 36 & 21 & 25 & 219 \\
\hline $\mathrm{V} 3$ & 0 & 2 & 2 & 1 & 0 & 0 & 0 & 0 & 0 & 1 & 7 & 3 & 16 \\
\hline \multicolumn{14}{|c|}{ SUB-BACIA 45} \\
\hline Classes do SPI & JAN & FEV & MAR & $\mathrm{ABR}$ & MAI & JUN & JUL & AGO & SET & OUT & NOV & DEZ & DS MESES \\
\hline $\mathrm{A} 3$ & 1 & 1 & 0 & 3 & 8 & 9 & 9 & 9 & 4 & 2 & 3 & 5 & 54 \\
\hline $\mathrm{A} 2$ & 43 & 57 & 33 & 45 & 38 & 24 & 64 & 29 & 46 & 37 & 43 & 32 & 491 \\
\hline A 1 & 62 & 47 & 73 & 54 & 46 & 193 & 298 & 247 & 61 & 71 & 56 & 65 & 1273 \\
\hline $\mathrm{N}$ & 170 & 153 & 188 & 171 & 220 & 174 & 29 & 115 & 276 & 167 & 170 & 176 & 2009 \\
\hline V1 & 58 & 73 & 34 & 57 & 88 & 0 & 0 & 0 & 13 & 39 & 56 & 62 & 480 \\
\hline $\mathrm{V} 2$ & 59 & 66 & 70 & 70 & 0 & 0 & 0 & 0 & 0 & 84 & 71 & 54 & 474 \\
\hline V3 & 7 & 3 & 2 & 0 & 0 & 0 & 0 & 0 & 0 & 0 & 1 & 6 & 19 \\
\hline
\end{tabular}


SUB-BACIA 46

\begin{tabular}{|c|c|c|c|c|c|c|c|c|c|c|c|c|c|}
\hline Classes do SPI & JAN & FEV & MAR & ABR & MAI & JUN & JUL & AGO & SET & OUT & NOV & DEZ & $\sum$ DOS MESES \\
\hline A 3 & 1 & 7 & 5 & 3 & 11 & 13 & 14 & 12 & 10 & 1 & 2 & 10 & 89 \\
\hline A2 & 67 & 63 & 51 & 70 & 61 & 50 & 123 & 122 & 64 & 73 & 66 & 54 & 864 \\
\hline A1 & 92 & 81 & 101 & 87 & 66 & 324 & 402 & 407 & 71 & 93 & 91 & 75 & 1890 \\
\hline N & 248 & 256 & 274 & 254 & 310 & 213 & 61 & 59 & 401 & 231 & 271 & 269 & 2847 \\
\hline V1 & 92 & 87 & 66 & 74 & 145 & 0 & 0 & 0 & 54 & 98 & 81 & 100 & 797 \\
\hline V2 & 92 & 97 & 92 & 110 & 7 & 0 & 0 & 0 & 0 & 102 & 74 & 82 & 656 \\
\hline V3 & 8 & 8 & 10 & 2 & 0 & 0 & 0 & 0 & 0 & 2 & 15 & 10 & 55 \\
\hline
\end{tabular}

\begin{tabular}{|l|l|l|l|l|l|l|l|l|l|l|l|l|l|}
\hline Classes do SPI & JAN & FEV & MAR & ABR & MAI & JUN & JUL & AGO & SET & OUT & NOV & DEZ & $\sum$ DOS MESES \\
\hline
\end{tabular}

\begin{tabular}{|c|c|c|c|c|c|c|c|c|c|c|c|c|c|}
\hline $\mathrm{A} 3$ & 2 & 0 & 1 & 1 & 2 & 4 & 4 & 2 & 2 & 3 & 0 & 3 & 24 \\
\hline $\mathrm{A} 2$ & 11 & 19 & 11 & 13 & 15 & 6 & 45 & 47 & 10 & 11 & 17 & 10 & 215 \\
\hline $\mathrm{A} 1$ & 9 & 10 & 18 & 17 & 7 & 44 & 6 & 11 & 48 & 14 & 14 & 14 & 212 \\
\hline $\mathrm{N}$ & 58 & 47 & 54 & 48 & 71 & 50 & 65 & 60 & 60 & 77 & 49 & 50 & 689 \\
\hline $\mathrm{V} 1$ & 21 & 21 & 15 & 28 & 25 & 10 & 0 & 0 & 0 & 15 & 21 & 19 & 175 \\
\hline $\mathrm{V} 2$ & 19 & 23 & 21 & 13 & 0 & 6 & 0 & 0 & 0 & 0 & 19 & 24 & 125 \\
\hline $\mathrm{V} 3$ & 0 & 0 & 0 & 0 & 0 & 0 & 0 & 0 & 0 & 0 & 0 & 0 & 0 \\
\hline \multicolumn{10}{|c|}{}
\end{tabular}

SUB-BACIA 48

\begin{tabular}{|l|l|l|l|l|l|l|l|l|l|l|l|l|l|}
\hline Classes do SPI & JAN & FEV & MAR & ABR & MAI & JUN & JUL & AGO & SET & OUT & NOV & DEZ & $\sum$ DOS MESES \\
\hline
\end{tabular}

\begin{tabular}{|c|c|c|c|c|c|c|c|c|c|c|c|c|c|}
\hline A3 & 11 & 4 & 1 & 4 & 5 & 11 & 9 & 8 & 10 & 7 & 7 & 6 & 83 \\
\hline A2 & 32 & 45 & 45 & 41 & 43 & 32 & 35 & 41 & 31 & 37 & 41 & 38 & 461 \\
\hline A1 & 48 & 57 & 65 & 58 & 50 & 46 & 60 & 73 & 176 & 51 & 43 & 57 & 784 \\
\hline N & 173 & 167 & 168 & 164 & 179 & 200 & 201 & 226 & 183 & 305 & 187 & 155 & 2308 \\
\hline V1 & 76 & 53 & 50 & 72 & 88 & 71 & 53 & 52 & 0 & 0 & 110 & 109 & 734 \\
\hline V2 & 59 & 72 & 66 & 60 & 35 & 40 & 42 & 0 & 0 & 0 & 12 & 35 & 421 \\
\hline V3 & 1 & 2 & 4 & 1 & 0 & 0 & 0 & 0 & 0 & 0 & 0 & 0 & 8 \\
\hline \multicolumn{10}{|c|}{ SUB-BACIA 49 }
\end{tabular}

\begin{tabular}{|l|l|l|l|l|l|l|l|l|l|l|l|l|l|}
\hline Classes do SPI & JAN & FEV & MAR & ABR & MAI & JUN & JUL & AGO & SET & OUT & NOV & DEZ & $\sum$ DOS MESES \\
\hline
\end{tabular}

\begin{tabular}{|c|c|c|c|c|c|c|c|c|c|c|c|c|c|}
\hline A3 & 5 & 0 & 1 & 1 & 3 & 3 & 3 & 2 & 3 & 0 & 1 & 2 & 24 \\
\hline A2 & 10 & 22 & 16 & 20 & 12 & 17 & 14 & 21 & 18 & 20 & 18 & 15 & 203 \\
\hline A1 & 17 & 19 & 26 & 27 & 25 & 18 & 16 & 17 & 22 & 20 & 22 & 23 & 252 \\
\hline N & 77 & 66 & 61 & 60 & 69 & 71 & 81 & 66 & 62 & 87 & 76 & 63 & 839 \\
\hline V1 & 33 & 24 & 25 & 23 & 19 & 23 & 21 & 25 & 36 & 22 & 37 & 50 & 338 \\
\hline V2 & 18 & 29 & 31 & 28 & 30 & 25 & 22 & 29 & 18 & 11 & 6 & 7 & 254 \\
\hline V3 & 0 & 0 & 0 & 1 & 2 & 3 & 3 & 0 & 1 & 0 & 0 & 0 & 10 \\
\hline
\end{tabular}

\begin{tabular}{|c|c|c|c|c|c|c|}
\hline \multicolumn{7}{|c|}{ LEGENDA } \\
\hline A3 & A2 & Al & N & V1 & V2 & V3 \\
\hline Extremamente Úmido & Muito Úmido & Moderadamente Úmido & Normal & Moderadamente Seco & Muito Seco & Extremamente Seco \\
\hline
\end{tabular}

Fonte: Organizado pelos autores 
Quadro 6. Número de Ocorrências Mensais por Classes do RAI por Sub-bacia

\begin{tabular}{|c|c|c|c|c|c|c|c|c|c|c|c|c|c|}
\hline \multicolumn{14}{|c|}{ SUB-BACIA 40} \\
\hline Classes do RAI & JAN & FEV & MAR & ABR & MAI & JUN & JUL & $\mathrm{AGO}$ & SET & OUT & NOV & DEZ & DOS MESES \\
\hline $\mathrm{C} 3$ & 65 & 76 & 75 & 79 & 87 & 112 & 113 & 91 & 70 & 84 & 84 & 63 & 999 \\
\hline $\mathrm{C} 2$ & 238 & 211 & 203 & 163 & 173 & 118 & 119 & 159 & 184 & 176 & 166 & 219 & 2129 \\
\hline $\mathrm{C} 1$ & 367 & 391 & 387 & 401 & 366 & 223 & 209 & 256 & 443 & 391 & 443 & 409 & 4286 \\
\hline S1 & 415 & 394 & 443 & 398 & 408 & 323 & 234 & 212 & 366 & 432 & 434 & 461 & 4520 \\
\hline S2 & 360 & 395 & 335 & 425 & 446 & 704 & 805 & 762 & 417 & 371 & 311 & 269 & 5600 \\
\hline S3 & 35 & 13 & 37 & 14 & 0 & 0 & 0 & 0 & 0 & 26 & 42 & 59 & 226 \\
\hline \multicolumn{14}{|c|}{ SUB-BACIA 41} \\
\hline Classes do RAI & JAN & FEV & MAR & ABR & MAI & JUN & JUL & AGO & SET & \begin{tabular}{|l|l|} 
OUT \\
\end{tabular} & NOV & DEZ & DOS MESES \\
\hline $\mathrm{C} 3$ & 48 & 45 & 49 & 56 & 48 & 64 & 58 & 58 & 48 & 51 & 51 & 51 & 627 \\
\hline $\mathrm{C} 2$ & 119 & 98 & 107 & 85 & 93 & 50 & 76 & 80 & 102 & 87 & 106 & 97 & 1100 \\
\hline $\mathrm{C} 1$ & 217 & 254 & 245 & 232 & 216 & 125 & 104 & 138 & 226 & 229 & 247 & 253 & 2486 \\
\hline S1 & 266 & 231 & 254 & 254 & 238 & 140 & 114 & 126 & 207 & 273 & 263 & 278 & 2644 \\
\hline S2 & 215 & 245 & 208 & 245 & 282 & 501 & 528 & 478 & 297 & 225 & 181 & 170 & 3575 \\
\hline S3 & 15 & 7 & 17 & 8 & 3 & 0 & 0 & 0 & 0 & 15 & 32 & 31 & 128 \\
\hline \multicolumn{14}{|c|}{ SUB-BACIA 42} \\
\hline Classes do RAI & JAN & FEV & MAR & ABR & MAI & JUN & JUL & $\mathrm{AGO}$ & SET & OUT & NOV & DEZ & DOS MESES \\
\hline $\mathrm{C} 3$ & 20 & 24 & 29 & 27 & 22 & 34 & 35 & 34 & 27 & 30 & 21 & 24 & 327 \\
\hline $\mathrm{C} 2$ & 71 & 61 & 58 & 53 & 61 & 33 & 16 & 33 & 42 & 48 & 68 & 58 & 602 \\
\hline $\mathrm{C} 1$ & 135 & 121 & 125 & 107 & 93 & 41 & 39 & 55 & 114 & 124 & 120 & 140 & 1214 \\
\hline S1 & 123 & 127 & 149 & 146 & 119 & 42 & 38 & 42 & 123 & 132 & 146 & 150 & 1337 \\
\hline S2 & 126 & 146 & 113 & 146 & 185 & 330 & 352 & 316 & 174 & 146 & 111 & 90 & 2235 \\
\hline S3 & 5 & 1 & 6 & 1 & 0 & 0 & 0 & 0 & 0 & 0 & 14 & 18 & 45 \\
\hline \multicolumn{14}{|c|}{ SUB-BACIA 43} \\
\hline Classes do RAI & JAN & FEV & MAR & $\mathrm{ABR}$ & MAI & JUN & JUL & AGO & SET & \begin{tabular}{|l|l|} 
OUT \\
\end{tabular} & NOV & DEZ & DOS MESES \\
\hline $\mathrm{C} 3$ & 12 & 12 & 9 & 14 & 12 & 18 & 14 & 15 & 14 & 13 & 12 & 12 & 157 \\
\hline C2 & 30 & 29 & 42 & 30 & 21 & 14 & 7 & 19 & 25 & 23 & 43 & 28 & 311 \\
\hline $\mathrm{C} 1$ & 72 & 60 & 59 & 57 & 44 & 24 & 6 & 24 & 54 & 71 & 65 & 62 & 598 \\
\hline S1 & 51 & 60 & 69 & 63 & 56 & 17 & 13 & 16 & 52 & 64 & 67 & 82 & 610 \\
\hline S2 & 74 & 78 & 59 & 75 & 107 & 167 & 200 & 166 & 95 & 69 & 42 & 46 & 1178 \\
\hline S3 & 1 & 1 & 2 & 1 & 0 & 0 & 0 & 0 & 0 & 0 & 11 & 10 & 26 \\
\hline \multicolumn{14}{|c|}{ SUB-BACIA 44} \\
\hline Classes do RAI & JAN & FEV & MAR & $\mathrm{ABR}$ & MAI & JUN & JUL & AGO & SET & \begin{tabular}{|l|l|} 
OUT \\
\end{tabular} & NOV & DEZ & DOS MESES \\
\hline $\mathrm{C} 3$ & 14 & 12 & 12 & 11 & 13 & 15 & 15 & 12 & 14 & 10 & 12 & 8 & 148 \\
\hline $\mathrm{C} 2$ & 16 & 20 & 22 & 28 & 15 & 7 & 5 & 9 & 13 & 26 & 26 & 21 & 208 \\
\hline $\mathrm{C} 1$ & 50 & 36 & 56 & 46 & 34 & 16 & 10 & 18 & 33 & 48 & 55 & 48 & 450 \\
\hline S1 & 55 & 67 & 57 & 42 & 39 & 9 & 5 & 12 & 43 & 57 & 63 & 73 & 522 \\
\hline S2 & 65 & 63 & 53 & 73 & 99 & 153 & 165 & 149 & 97 & 59 & 37 & 42 & 1055 \\
\hline S3 & 0 & 2 & 0 & 0 & 0 & 0 & 0 & 0 & 0 & 0 & 7 & 8 & 17 \\
\hline \multicolumn{14}{|c|}{ SUB-BACIA 45} \\
\hline Classes do RAI & JAN & FEV & MAR & ABR & MAI & JUN & JUL & AGO & SET & \begin{tabular}{|l|l|} 
OUT \\
\end{tabular} & NOV & DEZ & DOS MESES \\
\hline $\mathrm{C} 3$ & 28 & 26 & 23 & 27 & 25 & 25 & 22 & 25 & 26 & 20 & 18 & 20 & 285 \\
\hline $\mathrm{C} 2$ & 37 & 43 & 45 & 36 & 26 & 6 & 9 & 9 & 36 & 52 & 52 & 49 & 400 \\
\hline $\mathrm{C} 1$ & 105 & 72 & 121 & 102 & 59 & 30 & 34 & 29 & 72 & 92 & 125 & 110 & 951 \\
\hline S1 & 114 & 120 & 111 & 110 & 85 & 26 & 15 & 18 & 68 & 119 & 108 & 120 & 1014 \\
\hline $\mathrm{S} 2$ & 116 & 139 & 100 & 125 & 205 & 313 & 320 & 319 & 198 & 117 & 90 & 84 & 2126 \\
\hline S3 & 0 & 0 & 0 & 0 & 0 & 0 & 0 & 0 & 0 & 0 & 7 & 17 & 24 \\
\hline \multicolumn{14}{|c|}{ SUB-BACIA 46} \\
\hline Classes do RAI & JAN & FEV & MAR & ABR & MAI & JUN & JUL & AGO & SET & \begin{tabular}{|l|} 
OUT \\
\end{tabular} & NOV & DEZ & DOS MESES \\
\hline $\mathrm{C} 3$ & 35 & 38 & 31 & 36 & 44 & 36 & 36 & 41 & 39 & 35 & 33 & 29 & 433 \\
\hline $\mathrm{C} 2$ & 62 & 56 & 59 & 62 & 41 & 24 & 11 & 18 & 40 & 64 & 71 & 65 & 573 \\
\hline $\mathrm{C} 1$ & 165 & 145 & 159 & 149 & 100 & 45 & 27 & 24 & 117 & 155 & 174 & 159 & 1419 \\
\hline S1 & 162 & 193 & 202 & 184 & 154 & 32 & 33 & 31 & 95 & 158 & 182 & 194 & 1620 \\
\hline S2 & 171 & 162 & 140 & 168 & 261 & 463 & 493 & 486 & 309 & 188 & 115 & 136 & 3092 \\
\hline S3 & 5 & 6 & 9 & 1 & 0 & 0 & 0 & 0 & 0 & 0 & 25 & 17 & 63 \\
\hline
\end{tabular}


SUB-BACIA 47

\begin{tabular}{|c|c|c|c|c|c|c|c|c|c|c|c|c|c|}
\hline \multicolumn{14}{|c|}{ SUB-BACIA 47} \\
\hline Classes do RAI & JAN & FEV & MAR & $\mathrm{ABR}$ & MAI & JUN & JUL & AGO & SET & OUT & NOV & DEZ & $\sum$ DOS MESES \\
\hline $\mathrm{C} 3$ & 10 & 7 & 8 & 12 & 9 & 8 & 6 & 7 & 7 & 9 & 8 & 7 & 98 \\
\hline $\mathrm{C} 2$ & 3 & 14 & 7 & 6 & 8 & 2 & 5 & 5 & 8 & 5 & 12 & 7 & 82 \\
\hline $\mathrm{C} 1$ & 34 & 25 & 29 & 31 & 12 & 14 & 14 & 10 & 9 & 13 & 30 & 29 & 250 \\
\hline $\mathrm{S} 1$ & 27 & 33 & 39 & 29 & 28 & 17 & 13 & 9 & 12 & 21 & 31 & 36 & 295 \\
\hline S2 & 46 & 41 & 37 & 42 & 63 & 79 & 82 & 89 & 84 & 72 & 38 & 41 & 714 \\
\hline S3 & 0 & 0 & 0 & 0 & 0 & 0 & 0 & 0 & 0 & 0 & 1 & 0 & 1 \\
\hline \multicolumn{14}{|c|}{ SUB-BACIA 48} \\
\hline Classes do RAI & JAN & FEV & MAR & $\mathrm{ABR}$ & MAI & JUN & JUL & AGO & SET & OUT & NOV & DEZ & $\sum$ DOS MESES \\
\hline $\mathrm{C} 3$ & 21 & 21 & 22 & 25 & 26 & 26 & 20 & 22 & 27 & 29 & 24 & 20 & 283 \\
\hline $\mathrm{C} 2$ & 34 & 49 & 49 & 34 & 30 & 22 & 44 & 34 & 15 & 16 & 28 & 36 & 391 \\
\hline $\mathrm{C} 1$ & 85 & 109 & 101 & 106 & 87 & 78 & 79 & 65 & 49 & 67 & 79 & 91 & 996 \\
\hline S1 & 123 & 109 & 119 & 102 & 93 & 113 & 109 & 66 & 43 & 46 & 101 & 102 & 1126 \\
\hline S2 & 137 & 110 & 108 & 131 & 164 & 161 & 148 & 213 & 266 & 242 & 168 & 151 & 1999 \\
\hline S3 & 0 & 2 & 1 & 2 & 0 & 0 & 0 & 0 & 0 & 0 & 0 & 0 & 5 \\
\hline \multicolumn{14}{|c|}{ SUB-BACIA 49} \\
\hline Classes do RAI & JAN & FEV & MAR & $\mathrm{ABR}$ & MAI & JUN & JUL & AGO & SET & OUT & NOV & DEZ & $\sum$ DOS MESES \\
\hline $\mathrm{C} 3$ & 10 & 10 & 10 & 8 & 11 & 8 & 11 & 10 & 9 & 11 & 10 & 10 & 118 \\
\hline $\mathrm{C} 2$ & 6 & 16 & 17 & 17 & 14 & 20 & 11 & 20 & 14 & 13 & 16 & 12 & 176 \\
\hline $\mathrm{C} 1$ & 34 & 32 & 39 & 35 & 35 & 38 & 43 & 33 & 36 & 27 & 27 & 34 & 413 \\
\hline S1 & 50 & 51 & 41 & 53 & 55 & 53 & 57 & 54 & 45 & 33 & 34 & 40 & 566 \\
\hline S2 & 60 & 51 & 53 & 46 & 45 & 38 & 33 & 41 & 56 & 76 & 73 & 64 & 636 \\
\hline S3 & 0 & 0 & 0 & 1 & 0 & 3 & 5 & 2 & 0 & 0 & 0 & 0 & 11 \\
\hline
\end{tabular}

\begin{tabular}{|c|c|c|c|c|c|}
\hline \multicolumn{5}{|c|}{ LEGENDA } \\
\hline C3 & C2 & C1 & S1 & S2 & S3 \\
\hline Extremamente Úmido & Muito Úmido & Úmido & Seco & Muito Seco & Extremamente Seco \\
\hline
\end{tabular}

Fonte: Organizado pelos autores

\section{CONSIDERAÇÕES FINAIS}

O conhecimento do clima, em especial dos índices pluviométricos, é essencial para o acompanhamento da dinâmica do sistema natural e para a vida humana. Nesta perspectiva, estudos que aplicam ferramentas estatísticas buscando identificar possíveis tendências em tal variável são cada vez mais necessários frente às mudanças climáticas e suas consequências.

No presente artigo as três ferramentas utilizadas para análise de tendências (Regressão Linear, Teste de Mann-Kendall e Teste do Sinal) demonstraram resultados relativamente similares. Constatou-se uma concordância quanto à diminuição da pluviosidade na Região Hidrográfica do Rio São Francisco no intervalo de 1976-2015. Ressalta-se que os meses do ano que apresentaram significativas ocorrências de decréscimo chuvoso nos três métodos foram Janeiro, Fevereiro, Julho, Setembro, além do total anual. E que, em contrapartida, os meses com expressivas ocorrências de aumento na pluviosidade são Março e Novembro.

Quanto às ocorrências de extremos pluviométricos nos totais anuais e nos meses do ano, identificados através do SPI e do RAI, percebeu-se que elas estão presentes na série temporal utilizada e que há maior quantidade de ocorrências de anomalias positivas e negativas nas classes próximas ao normal e que vão diminuindo significativamente nas classes mais extremas.

Os resultados aqui identificados e demonstrados em relação às tendências e extremos pluviométricos na Região Hidrográfica do Rio São Francisco buscam contribuir e demonstrar possibilidades e necessidades de mais estudos sobre as mudanças climáticas, em especial, em escala regional/local. Esta pesquisa é uma forma de colaborar com outros trabalhos científicos sobre a temática e auxiliar as agendas políticas a promover mitigação e adaptação em relação aos possíveis 
impactos negativos que a diminuição dos índices pluviométricos e da maior possibilidade de ocorrências de episódios extremos podem acarretar.

\section{REFERÊNCIAS BIBLIOGRÁFICAS}

ABRAMOWITZ, M.; STEGUN, I. A. Handbook of mathematical functions with formulas, graphs, and mathematical tables. New York: Dover, 1965. $1046 \mathrm{p}$.

AGÊNCIA NACIONAL DE ÁGUAS - ANA. Disponível em Hidroweb - Sistema de Informações Hidrológicas: <http://www.snirh.gov.br/hidroweb/serieshistoricas $>$. Acesso em: 2017/2018/2019/2020.

ASSIS, J. M. O. de. Variabilidade do clima e cenários futuros de mudanças climáticas no trecho submédio da Bacia Hidrográfica do Rio São Francisco. Novembro de 2016. 187f. Tese de Doutorado (Doutorado em Engenharia Civil) Centro de Tecnologia e Geociências, Universidade Federal do Pernambuco - UFPE, Recife, nov. de 2016.

BACK, A. J. Aplicação de análise estatística para identificação de tendências climáticas. Revista Pesquisa Agropecuária Brasileira, Brasília, v. 36, n. 5, p. 717-726, 2001.

COMITÊ DA BACIA HIDROGRÁFICA DO RIO SÃO FRANCISCO - CBHSF (s.d.). Disponível em:<http://cbhsaofrancisco.org.br/a-bacia/>. Acesso em: jun. 2016.

FERNANDES, D.S.; HEINEMANN, A.B.; PAZ, R.L.F. da; AMORIM, A. de O.; CARDOSO, A.S. Índices para a quantificação da seca. Santo Antônio de Goiás: Embrapa Arroz e Feijão. Documentos, 244, 2009. 48 p.

FERREIRA, V. O. Análise de Tendências em Séries Pluviométricas: algumas Possibilidades Metodológicas. Revista Geonorte, Manacapuru/AM, Edição Especial 2, v. 1, n. 5, p. 317-324, 2012.

GARCIA, J. R.; MIYAMOTO, B. C.; MAIA, A. G. Eventos extremos de precipitação: identificação e análise da bacia hidrográfica do Rio Jundiaí, São Paulo. Revue FrancoBréslienne de Géographie, n. 37, 2018.

INSTITUTO BRASILEIRO DE GEOGRAFIA E ESTATÍSTICA - IBGE. Disponível em: $<$ https://www.ibge.gov.br/geociencias/downloads-geociencias.html>. Acesso em: 2017.

INTERGOVERNMENTAL PANEL ON CLIMATE CHANGE (IPCC). Managing the Risks of Extreme Events and Disasters to Advance Climate Change Adaptation (SREX), 2012. Disponível em: <http://ipcc-wg2.gov/SREX/images/uploads/SREXSPM_Approved-HiRes_opt.pdf.>. Acesso em nov. 2019.

INTERGOVERNMENTAL PANEL ON CLIMATE CHANGE (IPCC). Summary for Policymakers. In.: Climate Change 2013: The Physical Science Basis. Contribution of Working Group I to the Fifth Assessment Report of the Intergovernmental Panel on Climate Change [Stocker, T. F.; D. Qin, G. K. Plattnet, M. Itignor, S. K. Allen, I. 
Boschung, A. Nauels, Y. Xia, V. Bex and P. M. Midgley (eds.)]. Cambridge University Press, Cambridge, United Kingdom and New York, NY, USA, 2013.

INTERGOVERNMENTAL PANEL ON CLIMATE CHANGE (IPCC). Cambio climático 2014: Informe de síntesis. Contribución de los Grupos de Trabajo I, II y III al Quinto Informe de Evaluación del Grupo Intergubernamental de Expertos sobre el Cambio Climático [Equipo principal de redacción, R. K. Pachauri y L. A. Meyer (eds.)]. IPCC, Ginebra, Suiza, 2014, 157p.

MARENGO, J. A.; NOBRE, C. A.; SALATI, E.; AMBRIZZI, T. Caracterização do clima atual e definição das alterações climáticas para o território brasileiro ao longo do século XXI. Brasília: Ministério do Meio Ambiente (MMA), 2007, 54p.

McKee,T. B.; Doesken, N. J.; Kleist, J. The relationship of drought frequency and duration to times scale. In: Conference on Appied Climatology, 8.,1993, Boston. Anais American Meteorological Society. Boston: Preprints, p. 179-184, 1993.

MORETTIN, P. A.; TOLOI, C.M.C. Análise de Séries Temporais. - 2. ed. - São Paulo: Editora Blucher, 2006.

ROOY, M. P.V. A Rainfall Anomaly Index Independent of Time and Space. Notes, v.14, p.43, 1965.

SALVIANO, M. F.; GROPPO, J. D.; PELLEGRINO, G. Q. Análise de tendências em dados de precipitação e temperatura no Brasil. Revista Brasileira de Meteorologia, v. 31 , n. 1, p. 64-73, 2016.

SILVA, G. B.; SOUZA, W. M.; AZEVEDO, P. V. Cenários de mudanças climáticas no estado da Bahia através de estudos numéricos e estatísticos. Revista Brasileira de Geografia Física, Recife/PE, v. 05, n. 05, p. 1019-1034, 2012.

https://doi.org/10.26848/rbgf.v5i5.232891

SILVA, M. M.; FERREIRA, V. de O. Análise comparativa do clima de Araçuaí, Pedra Azul e Itamarandiba, na porção mineira da bacia do rio Jequitinhonha. Revista Caderno de Geografia, Belo Horizonte/MG, v. 21, n. 35, p. 56-73, jan./jun. 2011.

SILVEIRA, C. da S.; SOUZA FILHO, F. de A. de; MARTINS, E. S. P. R.; OLIVEIRA, J. L.; COSTA, A. C.; NOBREGA, M. T.; SOUZA, S. A. de; SILVA, R. F. V. Mudanças climáticas na bacia do rio São Francisco: uma análise para precipitação e temperatura. Revista Brasileira de Recursos Hídricos, Porto Alegre/RS, v. 21, n. 02, p. 416-428, 2016.

https://doi.org/10.21168/rbrh.v21n2.p416-428

SOARES, D. B.; NOBREGA, R. S.; GALVÍNCIO, J. D. Indicadores Climáticos de desertificação na bacia hidrográfica do rio Pajéu, Pernambuco. Revista Brasileira de Climatologia, Curitiba/PR, ano 14, v. 22, p. 363-380, jan./jun. 2018.

THOM, H. C. S. Some methods of climatological analysis. Geneva: World Meteorological Organization, 1966. 53 p. (WMO. Technical note, 81). 